\title{
STABLE UPWIND SCHEMES FOR THE MAGNETIC INDUCTION EQUATION*
}

\author{
Franz G. Fuchs ${ }^{1}$, Kenneth H. Karlsen ${ }^{1}$, Siddharta Mishra ${ }^{1}$ \\ AND NiLS H. RisEBRO ${ }^{1}$
}

\begin{abstract}
We consider the magnetic induction equation for the evolution of a magnetic field in a plasma where the velocity is given. The aim is to design a numerical scheme which also handles the divergence constraint in a suitable manner. We design and analyze an upwind scheme based on the symmetrized version of the equations in the non-conservative form. The scheme is shown to converge to a weak solution of the equations. Furthermore, the discrete divergence produced by the scheme is shown to be bounded. We report several numerical experiments that show that the stable upwind scheme of this paper is robust.
\end{abstract}

Mathematics Subject Classification. 65M12, 35L65.

Received December 6, 2007. Revised October 26, 2008.

Published online April 8, 2009.

\section{INTRODUCTION}

\subsection{The Model}

In this paper, we study the magnetic induction equation

$$
\partial_{t} \mathbf{B}+\operatorname{curl}(\mathbf{B} \times \mathbf{u})=0,
$$

where the unknown $\mathbf{B}=\mathbf{B}(\mathbf{x}, t) \in \mathbb{R}^{3}$ describes the magnetic field of a plasma in three space dimensions with coordinate $\mathbf{x}=(x, y, z)$. The above equation models the evolution of the magnetic field in the plasma which is moving with a prescribed velocity field $\mathbf{u}(\mathbf{x}, t)$. An immediate consequence of (1.1) is that the divergence of $\mathbf{B}$ is preserved in time, i.e.,

$$
\partial_{t}(\operatorname{div} \mathbf{B})=0 .
$$

Thus if the divergence is initially zero, it remains so.

Equation (1.1) is augmented with suitable initial and boundary conditions. In this paper, we focus on the Cauchy problem with the initial conditions

$$
\mathbf{B}(\mathbf{x}, 0)=\mathbf{B}_{0}(\mathbf{x}), \quad \mathbf{x} \in \mathbb{R}^{3} .
$$

Keywords and phrases. Conservation laws, induction equation, divergence constraint, upwinded source terms.

* This work was supported by the Research Council of Norway through an Outstanding Young Investigators Award of K.H. Karlsen.

1 Centre of Mathematics for Applications (CMA), University of Oslo, P.O. Box 1053, Blindern, 0316 Oslo, Norway.

franzf@math.uio.no; kennethk@math.uio.no; siddharm@cma.uio.no; nilshr@math.uio.no 
If we write $\mathbf{B}=\left(B^{1}, B^{2}, B^{3}\right)^{t}$ and $\mathbf{u}=\left(u^{1}, u^{2}, u^{3}\right)^{t}$, (1.1) reads

$$
\begin{aligned}
& \partial_{t} B^{1}-\partial_{y}\left(u^{1} B^{2}-u^{2} B^{1}\right)+\partial_{z}\left(u^{3} B^{1}-u^{1} B^{3}\right)=0, \\
& \partial_{t} B^{2}+\partial_{x}\left(u^{1} B^{2}-u^{2} B^{1}\right)-\partial_{z}\left(u^{2} B^{3}-u^{3} B^{2}\right)=0, \\
& \partial_{t} B^{3}-\partial_{x}\left(u^{3} B^{1}-u^{1} B^{3}\right)+\partial_{y}\left(u^{2} B^{3}-u^{3} B^{2}\right)=0 .
\end{aligned}
$$

For vectors $\mathbf{a}$ and $\mathbf{b}$ we use the notation

$$
\mathbf{a} \otimes \mathbf{b}=\left(\begin{array}{ccc}
a^{1} b^{1} & a^{1} b^{2} & a^{1} b^{3} \\
a^{2} b^{1} & a^{2} b^{2} & a^{2} b^{3} \\
a^{3} b^{1} & a^{3} b^{2} & a^{3} b^{3}
\end{array}\right) .
$$

Then we can rewrite (1.1) in conservative form

$$
\partial_{t} \mathbf{B}+\operatorname{div}(\mathbf{u} \otimes \mathbf{B}-\mathbf{B} \otimes \mathbf{u})=0,
$$

where the divergence of the matrix is the vector obtained by taking the divergence of the rows. The equation (1.1) can be derived from the full Maxwell's equations for electromagnetic fields by using the standard Lorentz transformations and the assumptions that the electric field at rest for a plasma is zero and that the plasma is a perfect conductor (so that we neglect the viscous terms). The details of the derivation of (1.1) can be found in any standard book on electrodynamics, for example [10]. One of the key issues in the design of numerical schemes for (1.1) is to handle the divergence constraint (1.2), i.e., to ensure that some discrete version of (1.2) holds at least approximately.

Equation (1.1) arises in a wide variety of contexts in the electrodynamics of plasmas. One important application is the equations of magnetohydrodynamics (MHD). MHD models the motion of a plasma in a magnetic field. In this case, the Euler equations of compressible gas dynamics are coupled with (1.1), see [13] for details. Numerical methods for MHD must address the divergence constraint, and several methods have been proposed in order to handle this constraint. These methods are in turn based on methods which preserve some discrete form of the divergence of $\mathbf{B}$.

In general, good numerical schemes for (1.1) is a step in the design of efficient numerical schemes for MHD, and a good motivation for studying (1.1) numerically. Our aim in this paper is to design, analyze and implement a simple upwind scheme for (1.1) and show that it is stable for very general initial data and velocity fields. In addition, the scheme also keeps divergence errors bounded and leads to sharp resolution of discontinuities. We start with a description of the continuous problem.

\subsection{The continuous problem}

In general, (1.1) is a system of linear conservation laws in three dimensions, it is hyperbolic, but not strictly hyperbolic.

In order to show existence of solutions to (1.1), we need to derive a priori estimates. The standard procedure for hyperbolic equations in multi space dimensions is to symmetrize the system and derive an energy estimate. In order to do this we introduce the operator

$$
\mathbf{a} \cdot \nabla=a^{1} \partial_{x}+a^{2} \partial_{y}+a^{3} \partial_{z},
$$

and write

$$
\begin{aligned}
\operatorname{curl}(\mathbf{B} \times \mathbf{u}) & =\mathbf{B} \operatorname{div} \mathbf{u}-\mathbf{u} \operatorname{div} \mathbf{B}+(\mathbf{u} \cdot \nabla) \mathbf{B}-(\mathbf{B} \cdot \nabla) \mathbf{u} \\
& =\left(u^{1} \mathbf{B}\right)_{x}+\left(u^{2} \mathbf{B}\right)_{y}+\left(u^{3} \mathbf{B}\right)_{z}-\mathbf{u} \operatorname{div} \mathbf{B}-(\mathbf{B} \cdot \nabla) \mathbf{u} .
\end{aligned}
$$


Thus (1.1) can also be recast as

$$
\partial_{t} \mathbf{B}+\left(u^{1} \mathbf{B}\right)_{x}+\left(u^{2} \mathbf{B}\right)_{y}+\left(u^{3} \mathbf{B}\right)_{z}=\mathbf{u d i v} \mathbf{B}+(\mathbf{B} \cdot \nabla) \mathbf{u}
$$

Then we see that a simple way to symmetrize (1.1) is to add a "source" term: (which is supposed to be zero anyway!) - udivB, resulting in

$$
\partial_{t} \mathbf{B}+\operatorname{curl}(\mathbf{B} \times \mathbf{u})=-\mathbf{u d i v} \mathbf{B} .
$$

Rewriting this, we find

$$
\begin{aligned}
\partial_{t} \mathbf{B}+(\mathbf{u} \cdot \nabla) \mathbf{B} & =-\mathbf{B}(\operatorname{div} \mathbf{u})+(\mathbf{B} \cdot \nabla) \mathbf{u} \\
& =M(D \mathbf{u}) \mathbf{B}
\end{aligned}
$$

where the matrix $M(D \mathbf{u})$ is given by

$$
M(D \mathbf{u})=\left(\begin{array}{ccc}
-\partial_{y} u^{2}-\partial_{z} u^{3} & \partial_{y} u^{1} & \partial_{z} u^{1} \\
\partial_{x} u^{2} & -\partial_{x} u^{1}-\partial_{z} u^{3} & +\partial_{z} u^{2} \\
\partial_{x} u^{3} & \partial_{y} u^{3} & -\partial_{x} u^{1}-\partial_{y} u^{2}
\end{array}\right)
$$

The above source term was first introduced for the non-linear MHD equations by Godunov in [9], see also [2,14]. This strategy of using a source term to handle constraints is very general and can be used for similar hyperbolic models involving other restrictions.

Let us write (1.4) as

$$
\partial_{t} \mathbf{B}+\partial_{x}\left(u^{1} \mathbf{B}\right)+\partial_{y}\left(u^{2} \mathbf{B}\right)+\partial_{z}\left(u^{3} \mathbf{B}\right)=(\mathbf{B} \cdot \nabla) \mathbf{u} .
$$

Introducing the matrices $A^{i}=u^{i} I$ for $i=1,2,3$, and

$$
C=-\left(\begin{array}{ccc}
\partial_{x} u^{1} & \partial_{y} u^{1} & \partial_{z} u^{1} \\
\partial_{x} u^{2} & \partial_{y} u^{2} & \partial_{z} u^{2} \\
\partial_{x} u^{3} & \partial_{y} u^{3} & \partial_{z} u^{1}
\end{array}\right)
$$

we may further rewrite (1.6) as

$$
\partial_{t} \mathbf{B}+\partial_{x}\left(A^{1} \mathbf{B}\right)+\partial_{y}\left(A^{2} \mathbf{B}\right)+\partial_{z}\left(A^{3} \mathbf{B}\right)+C \mathbf{B}=0 .
$$

This system is symmetric in the sense of Friedrichs (the matrices $A^{i}$ are symmetric). Regarding the functions $u^{1}, u^{2}$ and $u^{3}$ we assume that they are "sufficiently differentiable", i.e., whenever a derivative of $u^{i}$ is appearing in our calculations, we assume that this is a continuous and bounded function. Of course, this means that the matrices $A^{i}$ are also sufficiently smooth.

Observe that the above system is a special case of the more general problem

$$
\partial_{t} \mathbf{v}+\sum_{i=1}^{d} \Theta^{i}(\mathbf{x}, t) \partial_{x_{i}} \mathbf{v}=\Gamma(\mathbf{x}, t) \mathbf{v}+\mathbf{f}(\mathbf{x}, t), \quad \mathbf{v}(\mathbf{x}, 0)=\mathbf{v}_{0}(\mathbf{x})
$$

where the $d \times d$ matrices $\Theta^{1}, \ldots, \Theta^{d}$, and $B$ depend smoothly on $\mathbf{x}$ and $t$. We recall that the system (1.7) is Friedrichs symmetric if there exists a matrix $S_{0}(\mathbf{x}, t) \in C^{\infty}\left(\mathbb{R}^{d} \times \mathbb{R}\right)$ that is symmetric and uniformly positive definite, and the matrices

$$
S_{0}(\mathbf{x}, t) \Theta^{1}(\mathbf{x}, t), \ldots, S_{0}(\mathbf{x}, t) \Theta^{d}(\mathbf{x}, t)
$$

are symmetric for all $(\mathbf{x}, t)$ (see [3]). To analyze (1.7) one uses pseudo-differential calculus. We have the following well-posedness result, see [3], Theorem 2.6. 
Theorem 1.1. Suppose (1.7) has smooth coefficients and is Friedrichs symmetrizable. Fix $T>0$ and $s>1$. Assume that $f \in L^{2}\left(0, T ; H^{s}\left(\mathbb{R}^{d}\right)\right)$ and $v_{0} \in H^{s}\left(\mathbb{R}^{d}\right)$. Then there exists a unique (weak) solution $\mathbf{v} \in$ $C\left([0, T] ; H^{s}\left(\mathbb{R}^{d}\right)\right) \cap C^{1}\left([0, T] ; H^{s-1}\left(\mathbb{R}^{d}\right)\right)$ of the Cauchy problem (1.7). Moreover, there exists a constant, which is independent of the vector $v$, such that for any $t \in[0, T]$

$$
\|\mathbf{v}(\cdot, t)\|_{H^{s}\left(\mathbb{R}^{d}\right)}^{2} \leq C\left(\left\|\mathbf{v}_{0}\right\|_{H^{s}\left(\mathbb{R}^{d}\right)}^{2}+\int_{0}^{t}\|\mathbf{f}(\cdot, \tau)\|_{H^{s}\left(\mathbb{R}^{d}\right)}^{2} \mathrm{~d} \tau\right) .
$$

If $\mathbf{f} \in C^{\infty}\left([0, T] ; H^{\infty}\left(\mathbb{R}^{d}\right)\right)$ and $\mathbf{v}_{0} \in H^{\infty}\left(\mathbb{R}^{d}\right)$, then the solution $\mathbf{v}$ belongs to $C^{\infty}\left([0, T] ; H^{\infty}\left(\mathbb{R}^{d}\right)\right)$.

In the special case of a constant velocity field, the above equations decouple and reduce to

$$
\partial_{t} \mathbf{B}+(\mathbf{u} \cdot \nabla) \mathbf{B}=0
$$

and in this case we have the exact solution

$$
\mathbf{B}(\mathbf{x}, t)=\mathbf{B}_{0}(\mathbf{x}-\mathbf{u} t)
$$

Clearly in this special case, the exact solution is actually $T V D$. In general, we do not expect the initial data to be smooth. This is particularly true in the case of MHD equations where the magnetic field can have discontinuities. As a consequence, we define weak solutions of (1.1) by

Definition 1.1 (weak solution). For all function $\mathbf{u} \in H^{1}\left(\mathbb{R}^{3}\right)$ we call a locally integrable function $\mathbf{B}$ a weak solution of (1.1) if for all smooth test functions $\Phi \in \mathcal{C}_{0}^{1}$, the following integral identity holds:

$$
\int_{0}^{\infty} \int_{\mathbb{R}^{n}} \mathbf{B} \Phi_{t}+\mathbf{B}(\mathbf{u} \cdot \nabla) \Phi \mathrm{d} \mathbf{x} \mathrm{d} t=\int_{\mathbb{R}^{n}} \mathbf{B}_{0}(x) \Phi(\mathbf{x}, 0) \mathrm{d} \mathbf{x}+\int_{0}^{\infty} \int_{\mathbb{R}^{n}}(\mathbf{B} \cdot \nabla) \mathbf{u} \Phi \mathrm{d} \mathbf{x} \mathrm{d} t .
$$

Hence, weak solutions of (1.1) are defined in terms of the Friedrichs form (1.6). The existence of these is a consequence of Theorem 1.1.

For simplicity we will concentrate on the two-dimensional case, i.e., $\partial_{z}=0$ and $u^{3}=0$. In this case (1.3) reads

$$
\begin{aligned}
& \partial_{t} B^{1}+\partial_{y}\left(u^{1} B^{2}-u^{2} B^{1}\right)=0, \\
& \partial_{t} B^{2}-\partial_{x}\left(u^{1} B^{2}-u^{2} B^{1}\right)=0, \\
& \partial_{t} B^{3}+\partial_{x}\left(u^{1} B^{3}\right)+\partial_{y}\left(u^{2} B^{3}\right)=0 .
\end{aligned}
$$

The third equation is independent of the first two, and is such that if $B^{3}(x, y, 0)=0$, then also $B^{3}(x, y, t)=0$. Hence we ignore (1.11) in the remainder of this paper.

\subsection{Numerical schemes}

From the theory for the continuous problem, it is reasonable to require the following properties of a "good" numerical scheme for (1.1),

(i) The scheme should be upwind i.e., it should be able to resolve discontinuities in the solution sharply even at first order.

(ii) The scheme should be stable in the energy norm for a large class of initial data and velocity fields. 
(iii) In the special case of constant velocity fields, the scheme should be TVD.

(iv) Discrete versions of the divergence constraint should hold, at least approximately.

We shall consider first order schemes, since higher order extensions can be made once an efficient first order scheme is available. Note that the second property is essential for proving convergence, the third is a nonoscillatory property and the control of divergence is essential for respecting the constraint on (1.1).

Before we continue with a technical description of some schemes, let us introduce some useful notation. As usual, $\Delta x$ and $\Delta y$ denotes the spatial discretization parameters, these are (small) positive numbers. For $i$ and $j$ in $\mathbb{Z}$, let $x_{i}=i \Delta x, y_{j}=j \Delta y, x_{i+1 / 2}=x_{i}+\Delta x / 2$ and $y_{j+1 / 2}=y_{j}+\Delta y / 2$. Similarly we define the temporal discretization $\Delta t$, and set $t^{n}=n \Delta t$ for $n \geq 0$.

For a continuous function $a(x, y)$, we set

$$
a_{i, j}=a\left(x_{i}, y_{j}\right), \quad i \text { and } j \in \mathbb{Z} / 2 .
$$

For any quantity $\left\{a_{i, j}\right\}$ let the forward/backward differences be denoted by

$$
D_{x}^{ \pm} a_{i, j}= \pm \frac{a_{i \pm 1, j}-a_{i, j}}{\Delta x}, \quad D_{y}^{ \pm} a_{i, j}= \pm \frac{a_{i, j \pm 1}-a_{i, j}}{\Delta y}
$$

and the central differences

$$
D_{x, y}^{0}=\frac{1}{2}\left(D_{x, y}^{+}+D_{x, y}^{-}\right) .
$$

We also let the discrete time derivative be denoted by

$$
D_{t}^{+} b^{n}=\frac{b^{n+1}-b^{n}}{\Delta t}
$$

for any quantity $\left\{b^{n}\right\}_{n \geq 0}$. Furthermore, set $[a]^{+}=\max \{a, 0\}$ and $[a]^{-}=\min \{a, 0\}$. We shall need the following identities:

$$
\begin{aligned}
D_{x}^{ \pm}\left(a_{i} b_{i}\right) & =a_{i} D_{x}^{ \pm} b_{i}+b_{i \pm 1} D_{x}^{ \pm} a_{i} \\
D_{x}^{ \pm}\left(\left(a_{i}\right)^{2}\right) & =2 a_{i} D_{x}^{ \pm} a_{i} \pm \Delta x\left(D_{x}^{ \pm} a_{i}\right)^{2} \\
D_{x}^{0}\left(a_{j} b_{j}\right) & =a_{j} D_{x}^{0}\left(b_{j}\right)+b_{j} D_{x}^{0}\left(a_{j}\right)+\frac{\Delta x^{2}}{2}\left[\left(D_{x}^{+} a_{j}\right)\left(D_{x}^{-} D_{x}^{+} b_{j}\right)+\left(D_{x}^{-} b_{j}\right)\left(D_{x}^{-} D_{x}^{+} a_{j}\right)\right] \\
\sum_{i=I}^{J} a_{i}\left(D_{x}^{+} b_{i}\right) & =-\sum_{i=I+1}^{J}\left(D_{x}^{-} a_{i}\right) b_{i}+\frac{1}{\Delta x}\left(a_{J} b_{J+1}-a_{I} b_{I}\right) .
\end{aligned}
$$

We also let $I_{i j}$ denote the rectangle $\left(x_{i-1 / 2}, x_{i+1 / 2}\right] \times\left(y_{j-1 / 2}, y_{j+1 / 2}\right]$ and $I_{i, j}^{n}$ the cube $I_{i, j} \times[n \Delta t,(n+1) \Delta t)$.

When solving (1.10) numerically, we consider piecewise constant approximations

$$
\mathbf{B}_{i, j}^{n} \approx \frac{1}{\left|I_{i, j}\right|} \int_{I_{i, j}} \mathbf{B}\left(x, y, t^{n}\right) \mathrm{d} x \mathrm{~d} y
$$

in the fully discrete case, and

$$
\mathbf{B}_{i, j}(t) \approx \frac{1}{\left|I_{i, j}\right|} \int_{I_{i, j}} \mathbf{B}(x, y, t) \mathrm{d} x \mathrm{~d} y
$$



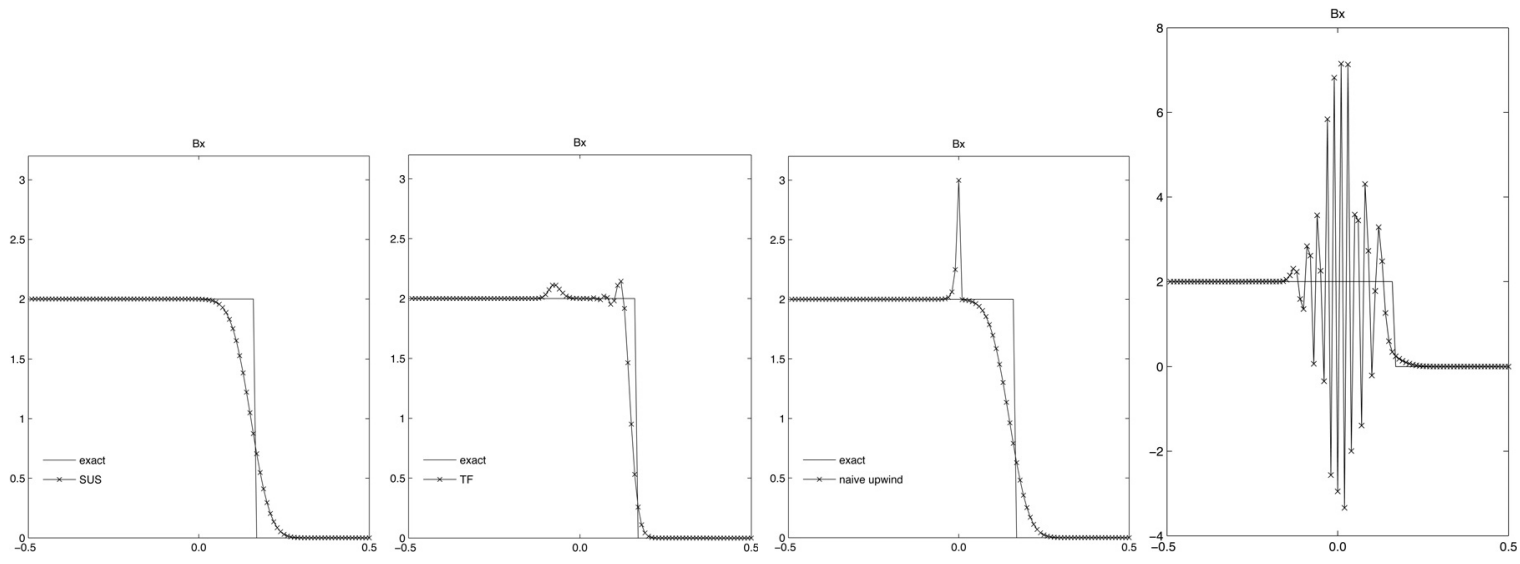

Figure 1. Approximations of $B^{1}(x, 0,0.3)$, initial data given by (3.1). From left to right: SUS, TF, standard upwind, central Powell.

for semi-discrete approximations. To obtain functions defined for all $\mathbf{x}$ and $t$, we define

$$
\begin{gathered}
\mathbf{B}^{\Delta t}(x, y, t)=\sum_{i, j} \mathbf{B}_{i, j}^{n} \mathbf{1}_{I_{i, j}^{n}}(x, y, t), \quad \text { and } \\
\mathbf{B}^{\Delta x}(x, y, t)=\sum_{i, j} \mathbf{B}_{i, j}(t) \mathbf{1}_{I_{i, j}}(x, y),
\end{gathered}
$$

where $\mathbf{1}_{\Omega}$ is the characteristic function of the set $\Omega$. All the schemes for (1.10) which we consider can be written as

$$
D_{t}^{+} \mathbf{B}_{i, j}^{n}=\mathcal{F}_{i, j}\left(\mathbf{u}, \mathbf{B}^{\Delta t}\left(\cdot, \cdot, t^{n}\right)\right) \quad \text { or } \quad \frac{\mathrm{d}}{\mathrm{d} t} \mathbf{B}_{i, j}(t)=\mathcal{F}_{i, j}\left(\mathbf{u}, \mathbf{B}^{\Delta x}(\cdot, \cdot, t)\right)
$$

for various functions $\mathcal{F}_{i, j}$

The system (1.1) contains terms of the type $\partial_{x}\left(u^{1} B^{2}\right)$, which we shall discretize in an upwind manner. To this end we introduce the notation:

$$
\begin{aligned}
D_{x}\{u, B\}_{i, j} & =D_{x}^{-}\left(\left[u_{i+1 / 2, j}\right]^{+} B_{i, j}\right)+D_{x}^{+}\left(\left[u_{i-1 / 2, j}\right]^{-} B_{i, j}\right) \\
& =\left[u_{j+1 / 2, j}\right]^{-} D_{x}^{+} B_{i, j}+\left[u_{i-1 / 2, j}\right]^{+} D_{x}^{-} B_{i, j}+B_{i, j} D_{x}^{-} u_{i+1 / 2, j} .
\end{aligned}
$$

An analogous expression defines $D_{y}\{u, B\}$. If $u$ and $B$ are smooth functions, then

$$
\partial_{x}(u B)\left(x_{i}, y_{j}\right)=D_{x}\{u, B\}_{i, j}+\mathcal{O}(\Delta x) .
$$

Equipped with above notation, the standard upwind scheme for (1.10) reads

$$
\begin{aligned}
& D_{t}^{+} B_{i, j}^{1, n}=-D_{y}\left\{u^{2}, B^{1, n}\right\}_{i, j}+D_{y}\left\{u^{1}, B^{2, n}\right\}_{i, j}, \\
& D_{t}^{+} B_{i, j}^{2, n}=-D_{x}\left\{u^{1}, B^{2, n}\right\}_{i, j}+D_{x}\left\{u^{2}, B^{1, n}\right\}_{i, j} .
\end{aligned}
$$

This scheme works well on many examples, but, it is not TVD when $\mathbf{u}$ is constant. As Example 3.1 and Figure 1 indicates, the scheme gives sharp oscillations near discontinuities in $\mathbf{B}$. In addition, the scheme does not have an energy estimate. Regarding the divergence constraint, the scheme does not preserve the central discrete divergence operator given by

$$
\operatorname{div}^{0}\left(\mathbf{B}_{i, j}\right)=D_{x}^{0}\left(B_{i, j}^{1}\right)+D_{y}^{0}\left(B_{i, j}^{2}\right) .
$$


In fact, the divergence errors can be very large in some numerical experiments. Since preserving the divergence constraint is a key numerical issue, several approaches have been suggested to couple upwinding of numerical schemes for (1.1) along with preservation/control of numerical divergence. The motivation behind most of these schemes is the need for efficient schemes for the MHD equations. A good review of divergence preserving schemes for MHD can be found in [20]. We now provide a very brief survey relevant to the situation considered here.

\subsubsection{Projection methods}

These methods are based on the Hodge decomposition of the magnetic field. At each time step, the magnetic field obtained from (1.17), denoted $\tilde{\mathbf{B}}^{n+1}$, can be written as

$$
\tilde{\mathbf{B}}^{n+1}=\operatorname{grad} \Psi+\operatorname{curl} \Phi \quad \Rightarrow \quad \Delta \Psi=\operatorname{div}\left(\tilde{\mathbf{B}}^{n+1}\right)
$$

Solving the last equation for $\Psi$ and then setting

$$
\mathbf{B}^{n+1}=\tilde{\mathbf{B}}^{n+1}-\operatorname{grad} \Psi
$$

makes $\mathbf{B}^{n+1}$ divergence free. The computational cost of this is significant, as one needs to solve an elliptic equation at each time step. This method was proposed in [5].

\subsubsection{Design of discrete divergence free operators/staggering}

Another strategy to control divergence errors is to use difference methods that preserve some (not necessarily $\operatorname{div}^{0}$ ) discrete form of the divergence. An important contribution in this direction is the paper of Torrilhon and Fey [19], where following scheme was proposed

$$
\begin{aligned}
& D_{t}^{+} B_{i, j}^{1, n}=\frac{1}{2}\left(D_{y}^{-}\left(\varphi_{i, j}^{1}+\varphi_{i-1, j}^{1}+\varphi_{i, j}^{2}+\varphi_{i+1, j}^{2}\right)+D_{y}^{+}\left(\varphi_{i, j}^{3}+\varphi_{i+1, j}^{3}+\varphi_{i, j}^{4}+\varphi_{i-1, j}^{4}\right)\right), \\
& D_{t}^{+} B_{i, j}^{2, n}=-\frac{1}{2}\left(D_{x}^{-}\left(\varphi_{i, j}^{1}+\varphi_{i, j-1}^{1}+\varphi_{i, j}^{4}+\varphi_{i, j+1}^{4}\right)+D_{x}^{+}\left(\varphi_{i, j}^{2}+\varphi_{i, j-1}^{2}+\varphi_{i, j}^{3}+\varphi_{i, j+1}^{3}\right)\right),
\end{aligned}
$$

where

and

$$
\varphi_{i, j}^{k}=\omega_{i, j}^{k}\left(u_{i, j}^{1} B_{i, j}^{2, n}-u_{i, j}^{2} B_{i, j}^{1, n}\right)
$$

$$
\begin{gathered}
\omega_{i, j}^{k}=\frac{\left[\mathbf{n}^{k} \cdot \mathbf{u}_{i, j}\right]^{+}}{\sum_{k=1}^{4}\left[\mathbf{n}^{k} \cdot \mathbf{u}_{i, j}\right]^{+}}, \\
\mathbf{n}^{1}=(1,1), \mathbf{n}^{2}=(-1,1), \mathbf{n}^{3}=(-1,-1) \text { and } \mathbf{n}^{4}=(1,-1) .
\end{gathered}
$$

This scheme preserves the following discrete divergence,

$$
\operatorname{div}^{*}\left(\mathbf{B}_{i, j}\right)=\frac{1}{4}\left(D_{x}^{0}\left(B_{i, j+1}^{1}+2 B_{i, j}^{1}+B_{i, j-1}^{1}\right)+D_{y}^{0}\left(B_{i+1, j}^{2}+2 B_{i, j}^{2}+B_{i-1, j}^{1}\right)\right) .
$$

If $\mathbf{B}$ is smooth, div ${ }^{*}$ differs from $\operatorname{div}^{0}$ by $\mathcal{O}\left(\Delta x^{2}+\Delta y^{2}\right)$, with a constant depending on the second derivatives of B. More details on this scheme can be found in $[18,19]$. In [19] this scheme is proved to be von Neumann stable if $\mathbf{u}$ is constant. However, we were unable to prove that it is stable in the energy norm when the velocity field varies in space and time. Also, the scheme is not TVD when the velocity field is constant, as is shown in Example 3.1 and Figure 1. Some numerical experiments in Section 3 show that even though the scheme preserves the discrete divergence div ${ }^{*}$, the central discrete divergence div $^{0}$ is not preserved and can be large.

In $[18,19]$ it was remarked that the scheme (1.20) is equivalent to staggering the discretizations of the velocity and magnetic fields. In this approach, the velocity and magnetic fields in the $x$-direction are centered on the cell edges in the $x$-direction and the velocity and magnetic fields in the $y$-direction are centered on the cell-edges 
in the $y$-direction. This approach has been proposed in number a papers including $[1,6,7,16,17]$ and details can be found in these references.

The main advantage of schemes based on this approach is the fact that some form of discrete divergence is preserved. Unfortunately, it is not possible to prove energy bounds (and hence convergence) for general nonconstant velocity fields. Furthermore, these schemes can be oscillatory near discontinuities as shown in some numerical examples in this paper.

\subsubsection{Schemes using the Godunov-Powell source term}

Another common approach to controlling the divergence, at least in the context of non-linear MHD equations, is to numerically solve (1.4) rather than (1.1). This approach was proposed by Powell in [14]. Formally, taking the divergence on both sides of (1.4)

$$
\partial_{t}(\operatorname{div}(\mathbf{B}))+(\mathbf{u} \cdot \nabla)(\operatorname{div} \mathbf{B})=-(\operatorname{div} \mathbf{u})(\operatorname{div} \mathbf{B})
$$

Hence, any non-zero divergence is advected along $\mathbf{u}$, and hopefully out of the computational domain.

Another key point about introducing the Godunov-Powell source term at the continuous level is to symmetrize (1.1) and write it in the form (1.6) which results in the derivation of energy estimates.

At the level of numerical schemes, one of the key issues is how to discretize the Godunov-Powell source term. It is common in the literature to use a discretization of the source term based on central differences (see, e.g., $[15])$.

Example 3.1 of this paper shows that such an approach might result in large oscillations in the solution and should be avoided. In [14], the author incorporates the source term into flux thus implicitly upwinding it.

Another way to upwind the Godunov-Powell source term is to discretize the Friedrichs form (1.6). In [4], the authors propose high order discontinuous Galerkin methods based on the "conservative" Friedrichs form (1.6) and show convergence results and error estimates for the scheme. They use locally divergence free basis functions, but they were unable to obtain any global divergence bound. The first order version the scheme proposed in [4] leads to an upwind discretization of the Godunov-Powell source term.

If the Friedrichs form is used as a basis for discretization of (1.1), one can appeal to the considerable literature that is devoted to finite volume schemes for Friedrichs systems. A notable reference in this regard is the work of Vila and Villedeau [21], and other related works of these authors. In [21], the authors analyze finite volume schemes for general Friedrichs systems and show convergence results and error estimates under very general assumptions. These results were further extended to even weaker solutions and error estimates by Jovanovič and Rohde in [11]. Thus, any finite volume scheme based on the Friedrichs form (1.6) falls into the framework of these papers.

Even though finite volume schemes based on the Friedrichs form (1.6) can be proved to converge and have error estimates for very general velocity fields, it has not been possible to derive bounds on the discrete divergence produced by these schemes. Heuristically, arguments of [14] imply that there is some control of divergence due to the fact that schemes should satisfy some discrete version of (1.21), and any divergence created by the scheme should be transported out of the domain. Yet, we have yet to find any rigorous proof of this fact. Even in [4], the authors were able to use local divergence free elements but were unable to control the divergence jump terms.

Another related problem with this approach, particularly in the context of the non-linear MHD equations, is the fact that (1.4) is not conservative. Hence, the Rankine-Hugoniot conditions are modified by the presence of the source term and this can lead to incorrect propagation speeds for strong shocks as pointed out in [14]. Other references attesting to this fact can be found in [20]. However, the errors are quite small. In case of the linear induction equation, we were unable to find any such errors at linear contact discontinuities in our numerical experiments.

Summing up, the two most common approaches to discretization of (1.1) are based on staggering/preserving some form of the discrete divergence, or on introducing the Godunov-Powell source term (the Friedrichs form of the equations). Using schemes based on staggering like in [19], one is able to preserve some form of discrete 
divergence exactly, but it is not possible to prove energy estimates (and hence convergence) for non-constant velocity fields. The resulting schemes can lead to oscillations near discontinuities even for constant velocity fields. On the other hand, use of the Friedrichs form or upwinding the Godunov-Powell source term leads to schemes for which we can prove energy estimates, convergence and even convergence rates. These schemes are non-oscillatory near discontinuities, at least for constant velocity fields. Yet, no rigorous control of the discrete divergence is available.

Given this, we propose a new class of finite difference schemes for (1.1). These schemes are based on the non-conservative form of the Friedrichs system (1.5). We use point values of the velocity field coupled with a upwind discretization of the transport terms. The sources in (1.5) are discretized by using central differences. Furthermore, we need to add some extra numerical diffusion at points where the velocity field vanishes. All these ingredients result in a scheme for which we are able to prove energy bounds, maximum principles and show that the scheme converges to a weak solution (1.9). In the case where the velocity field is constant, our scheme is $T V D$ and preserves any consistent discrete divergence operator. Most crucially, the upwind scheme of this paper has bounded discrete divergence i.e., we are able to show that the standard discrete divergence $\operatorname{div}^{0}$ produced by this scheme is bounded in $L^{2}$. Nevertheless, the resulting scheme is simple to implement.

Compared with other results, our scheme is in the spirit of schemes based on the symmetrized form of the equations. The key difference is that we discretize the non-conservative form of the symmetrized equations and have to add some extra (very small) numerical diffusion at sonic points (i.e., points where $|\mathbf{u}|=0$ ). This seems to be crucial to obtain bounds on the discrete divergence. We were unable to rewrite the upwind scheme of this paper in a form that falls directly into the class of schemes analyzed in [11,21] and hence, the general results of those papers do not apply to our scheme and the energy estimates need to proved independently. Numerical results obtained with the scheme show that the scheme is very robust, resolves the discontinuities well even at first order, and does not generate spurious oscillations around discontinuities. We are planning to use this upwind scheme for (1.1) in conjunction with suitable approximate Riemann solvers for the fluid part in order to design efficient splitting schemes for the MHD equations in a forthcoming paper [8].

The rest of this paper is organized as follows: in Section 2, we present the stable upwind scheme in two space dimensions, and prove stability and convergence. In Section 3, we present several numerical examples and compare the stable upwind scheme with other schemes.

\section{A STABle UPWIND SCHEME}

For simplicity, we restrict our presentation to two spatial dimensions. As stated in the introduction, we are going to discretize the nonconservative version of the equation (1.5), which in two dimensions takes the form

$$
\begin{aligned}
& \left(B^{1}\right)_{t}+u^{1}\left(B^{1}\right)_{x}+u^{2}\left(B^{1}\right)_{x}=-\left(u^{2}\right)_{y} B^{1}+\left(u^{1}\right)_{y} B^{2} \\
& \left(B^{2}\right)_{t}+u^{1}\left(B^{2}\right)_{x}+u^{2}\left(B^{2}\right)_{x}=\left(u^{2}\right)_{x} B^{1}-\left(u^{1}\right)_{x} B^{2} .
\end{aligned}
$$

Our scheme is initiated by setting

$$
\mathbf{B}_{i, j}^{0}=\frac{1}{\left|I_{i, j}\right|} \int_{I_{i, j}} \mathbf{B}_{0}(x, y) \mathrm{d} x \mathrm{~d} y .
$$

Regarding $\mathbf{u}$, we either assume that $\mathbf{u}$ is a continuous function of $x$ and $y$, or that we have given $\mathbf{u}$ as a table $\mathbf{u}_{i, j}$. The following definitions are also useful

$$
M_{i, j}=\left(\begin{array}{cc}
-D_{y}^{0} u_{i, j}^{2} & D_{y}^{0} u_{i, j}^{1} \\
D_{x}^{0} u_{i, j}^{2} & -D_{x}^{0} u_{i, j}^{1}
\end{array}\right),
$$


and

$$
\begin{aligned}
\left(\mathbf{u} \cdot D^{\mathrm{upw}}\right)_{i, j} & =\left[u_{i, j}^{1}\right]^{-} D_{x}^{+}+\left[u_{i, j}^{1}\right]^{+} D_{x}^{-}+\left[u_{i, j}^{2}\right]^{-} D_{y}^{+}+\left[u_{i, j}^{2}\right]^{+} D_{y}^{-} \\
\left(\mathbf{u} \cdot D^{0}\right)_{i, j} & =u_{i, j}^{1} D_{x}^{0}+u_{i, j}^{2} D_{y}^{0} \\
\sigma_{\delta}\left(\mathbf{u}_{i, j}\right)\left(\Delta_{\Delta y}^{\Delta x}\right) \cdot D^{2} & =\sigma_{\delta}\left(u_{i, j}^{1}\right) \Delta x D_{x}^{+} D_{x}^{-}+\sigma_{\delta}\left(u_{i, j}^{2}\right) \Delta y D_{y}^{+} D_{y}^{-},
\end{aligned}
$$

where the auxiliary function $\sigma_{\delta}$ is an even smooth function such that

$$
\sigma_{\delta}(a)= \begin{cases}\frac{\delta}{2}, & \text { if }|a| \leq \delta / 2, \\ 0, & \text { if }|a| \geq \delta .\end{cases}
$$

Furthermore, we demand that $\sigma$ is non-increasing in the interval $[0, \delta]$ and that $\left|\sigma_{\delta}^{\prime}(a)\right|<2$ for all $a$.

Then the numerical scheme in the fully discrete form is given by

$$
D_{t}^{+} \mathbf{B}_{i, j}^{n}+\left(\mathbf{u} \cdot D^{\mathrm{upw}}\right)_{i, j} \mathbf{B}_{i, j}^{n}=M_{i, j} \mathbf{B}_{i, j}^{n}+\sigma_{\delta}\left(\mathbf{u}_{i, j}\right)\left(\begin{array}{c}
\Delta x \\
\Delta y
\end{array}\right) \cdot D^{2} \mathbf{B}_{i, j}^{n}, \quad(i, j) \in \mathbb{Z}^{2}, n \geq 0 .
$$

The semi-discrete version of this reads

$$
\begin{aligned}
\frac{\mathrm{d}}{\mathrm{d} t} \mathbf{B}_{i, j}+\left(\mathbf{u} \cdot D^{\mathrm{upw}}\right)_{i, j} \mathbf{B}_{i, j} & =M_{i, j} \mathbf{B}_{i, j}+\sigma_{\delta}\left(\mathbf{u}_{i, j}\right)\left(\Delta_{\Delta y} x\right) \cdot D^{2} \mathbf{B}_{i, j}, \quad t>0, \\
\mathbf{B}_{i, j}(0) & =\mathbf{B}_{i, j}^{0}, \quad(i, j) \in \mathbb{Z}^{2} .
\end{aligned}
$$

The semi-discrete form is an infinite system of ordinary differential equations,

$$
\frac{\mathrm{d}}{\mathrm{d} t} \mathbf{B}=\mathcal{F}(\mathbf{B})
$$

where we can regard $\mathbf{B} \in \ell^{2} \times \ell^{2}$, and $\mathcal{F}: \ell^{2} \times \ell^{2} \rightarrow \ell^{2} \times \ell^{2}$ is given by

$$
(\mathcal{F}(\mathbf{B}))_{i, j}=-\left(\mathbf{u} \cdot D^{\mathrm{upw}}\right)_{i, j} \mathbf{B}_{i, j}+M_{i, j} \mathbf{B}_{i, j}+\sigma_{\delta}\left(\mathbf{u}_{i, j}\right)\left(\begin{array}{c}
\Delta x \\
\Delta y
\end{array}\right) \cdot D^{2} \mathbf{B}_{i, j} .
$$

For fixed $\Delta x$ and $\Delta y$, it is not difficult to show that

$$
\|\mathcal{F}(\mathbf{B})\|_{\ell^{2} \times \ell^{2}} \leq \frac{C}{\min \{\Delta x, \Delta y\}}\|\mathbf{B}\|_{\ell^{2} \times \ell^{2}} .
$$

Therefore $\mathcal{F}$ is Lipschitz continuous, and we have existence of a differentiable solution $\mathbf{B}(t)$ at least for small $t$. The energy bound, Lemma 2.1, ensures that we do not have any blow up in finite time. Therefore there exists a differentiable solution $\mathbf{B}(t)$ of $(2.7)$ for all $t>0$.

Componententwise, (2.6) takes the form

$$
\begin{aligned}
D_{t}^{+} B_{i, j}^{1, n}= & -\left[u_{i, j}^{1}\right]^{-} D_{x}^{+}\left(B_{i, j}^{1, n}\right)-\left[u_{i, j}^{1}\right]^{+} D_{x}^{-}\left(B_{i, j}^{1, n}\right)-\left[u_{i, j}^{2}\right]^{-} D_{y}^{+}\left(B_{i, j}^{1, n}\right)-\left[u_{i, j}^{2}\right]^{+} D_{y}^{-}\left(B_{i, j}^{1, n}\right) \\
& -D_{y}^{0}\left(u_{i, j}^{2}\right) B_{i, j}^{1, n}+D_{y}^{0}\left(u_{i, j}^{1}\right) B_{i, j}^{2, n}+\sigma_{\delta}\left(u_{i, j}^{1}\right) \Delta x D_{x}^{+} D_{x}^{-}\left(B_{i, j}^{1, n}\right)+\sigma_{\delta}\left(u_{i, j}^{2}\right) \Delta y D_{y}^{+} D_{y}^{-}\left(B_{i, j}^{1, n}\right), \\
D_{t}^{+} B_{i, j}^{2, n}= & -\left[u_{i, j}^{1}\right]^{-} D_{x}^{+}\left(B_{i, j}^{2, n}\right)-\left[u_{i, j}^{1}\right]^{+} D_{x}^{-}\left(B_{i, j}^{2, n}\right)-\left[u_{i, j}^{2}\right]^{-} D_{y}^{+}\left(B_{i, j}^{2, n}\right)-\left[u_{i, j}^{2}\right]^{+} D_{y}^{-}\left(B_{i, j}^{2, n}\right) \\
& +D_{x}^{0}\left(u_{i, j}^{2}\right) B_{i, j}^{1, n}-D_{x}^{0}\left(u_{i, j}^{1}\right) B_{i, j}^{2, n}+\sigma_{\delta}\left(u_{i, j}^{1}\right) \Delta x D_{x}^{+} D_{x}^{-}\left(B_{i, j}^{2, n}\right)+\sigma_{\delta}\left(u_{i, j}^{2}\right) \Delta y D_{y}^{+} D_{y}^{-}\left(B_{i, j}^{2, n}\right) .
\end{aligned}
$$

We remark that the scheme is based on a upwind discretization of the nonconservative symmetric form (2.1). In addition, to the upwind discretization we also need to add a small amount of explicit numerical diffusion at the sonic points. This is necessary in the subsequent analysis. 
Using the notation

$$
|a|_{\delta}=|a|+\sigma_{\delta}(a)
$$

and

$$
\left(|\mathbf{u}|_{\delta}\left(\begin{array}{c}
\Delta x \\
\Delta y
\end{array}\right) \cdot D^{2}\right)_{i, j}=\left|u_{i, j}^{1}\right|_{\delta} \Delta x D_{x}^{-} D_{x}^{+}+\left|u_{i, j}^{2}\right|_{\delta} \Delta y D_{y}^{-} D_{y}^{+},
$$

the scheme (2.6) can also be rewritten using central discrete derivatives

$$
D_{t}^{+} \mathbf{B}_{i, j}^{n}+\left(\mathbf{u} \cdot D^{0}\right)_{i, j} \mathbf{B}_{i, j}^{n}=M_{i, j} \mathbf{B}_{i, j}+\frac{1}{2}\left(|\mathbf{u}|_{\delta}\left(\begin{array}{c}
\Delta x \\
\Delta y
\end{array}\right) \cdot D^{2}\right)_{i, j} \mathbf{B}_{i, j}^{n} .
$$

The semi-discrete form of this is obtained replacing $D_{t}^{+}$by $\mathrm{d} / \mathrm{d} t$.

We are going to prove that both the fully discrete and the semi-discrete schemes have solutions that are bounded in both the energy and the maximum norms. In addition, we will also show that the semi-discrete scheme leads to a discrete divergence that is bounded in the $L^{2}$ norm. This is the main reason why we work with a discretization of the non-conservative form of the Friedrichs system (2.1).

In addition to the energy and divergence bounds, we are going to show that the scheme (2.8) converges to a weak solution (1.9) of the equation (1.6). To do this we need the "conservative", form i.e., the discrete form consistent with the two dimensional version of (1.6). Since we use point values of the coefficient $\mathbf{u}$ in our scheme this is not completely straightforward. Using the discrete Leibnitz rule for the central differences (1.14), we can rewrite $(2.8)$ as,

$$
\begin{aligned}
D_{t}^{+} B_{i, j}^{1, n}=- & D_{x}^{0}\left(u_{i, j}^{1} B_{i, j}^{1, n}\right)-D_{y}^{0}\left(u_{i, j}^{2} B_{i, j}^{1, n}\right)+D_{x}^{0}\left(u_{i, j}^{1}\right) B_{i, j}^{1, n}+D_{y}^{0}\left(u_{i, j}^{1}\right) B_{i, j}^{2, n}+\frac{1}{2}\left(|\mathbf{u}|_{\delta}\left(\begin{array}{c}
\Delta x \\
\Delta y
\end{array}\right) \cdot D^{2}\right)_{i, j} B_{i, j}^{1, n} \\
& +\frac{\Delta x^{2}}{2}\left[\left(D_{x}^{+} u_{i, j}^{1, n}\right)\left(D_{x}^{-} D_{x}^{+} B_{i, j}^{1}\right)+\left(D_{x}^{-} B_{i, j}^{1, n}\right)\left(D_{x}^{-} D_{x}^{+} u_{i, j}^{1}\right)\right] \\
& +\frac{\Delta y^{2}}{2}\left[\left(D_{y}^{+} u_{i, j}^{2}\right)\left(D_{y}^{-} D_{y}^{+} B_{i, j}^{1, n}\right)+\left(D_{y}^{-} B_{i, j}^{1, n}\right)\left(D_{y}^{-} D_{y}^{+} u_{i, j}^{2}\right)\right] \\
D_{t}^{+} B^{2, n}=- & D_{x}^{0}\left(u_{i, j}^{1} B_{i, j}^{2, n}\right)-D_{y}^{0}\left(u_{i, j}^{2} B_{i, j}^{2, n}\right)+D_{x}^{0}\left(u_{i, j}^{2}\right) B_{i, j}^{1, n}+D_{y}^{0}\left(u_{i, j}^{2}\right) B_{i, j}^{2, n}+\frac{1}{2}\left(|\mathbf{u}|_{\delta}\left(\begin{array}{c}
\Delta x \\
\Delta y
\end{array}\right) \cdot D^{2}\right)_{i, j} B_{i, j}^{2, n} \\
& +\frac{\Delta x^{2}}{2}\left[\left(D_{x}^{+} u_{i, j}^{1}\right)\left(D_{x}^{-} D_{x}^{+} B_{i, j}^{2, n}\right)+\left(D_{x}^{-} B_{i, j}^{2, n}\right)\left(D_{x}^{-} D_{x}^{+} u_{i, j}^{1}\right)\right] \\
& +\frac{\Delta y^{2}}{2}\left[\left(D_{y}^{+} u_{i, j}^{2}\right)\left(D_{y}^{-} D_{y}^{+} B_{i, j}^{2, n}\right)+\left(D_{y}^{-} B_{i, j}^{2, n}\right)\left(D_{y}^{-} D_{y}^{+} u_{i, j}^{2}\right)\right]
\end{aligned}
$$

Rewritten in the above form, (2.8) represents a consistent discretization of (1.6). Note that (2.10) and (2.11), we use conservative central differences, a stabilizing diffusion term and second order error terms due to the discrete Liebnitz rule.

We have not managed to write (2.8) as a special case of the class of finite volume schemes that were presented and analysed in [11,21]. The reason for this is the non-conservative form of the scheme. This is reflected in the presence of error terms in the conservative form (2.10) and (2.11) which have second derivatives in B multiplied by derivatives of $\mathbf{u}$, which can be of either sign. Hence, the results of $[11,21]$ do not apply directly to our scheme.

\subsection{Some estimates}

Now we prove that $\mathbf{B}^{\Delta t}$ satisfies some estimates which will allow us to conclude that $\left\{\mathbf{B}^{\Delta t}\right\}$ is weakly compact, and that any limit is a weak solution to (1.6). We start by

Lemma 2.1. Let $\left\{\mathbf{B}_{i, j}^{n}\right\}$ satisfy the scheme (2.6), and $\Delta t$ the following CFL-condition

$$
\Delta t \max \left\{\frac{6\left\|u^{1}\right\|_{L^{\infty}\left(\mathbb{R}^{2}\right)}}{\Delta x}, \frac{6\left\|u^{2}\right\|_{L^{\infty}\left(\mathbb{R}^{2}\right)}}{\Delta y}, 32 \delta\right\} \leq \frac{1}{2} .
$$


Then

$$
\Delta x \Delta y \sum_{i, j}\left|\mathbf{B}_{i, j}^{n}\right|^{2} \leq \mathrm{e}^{M n \Delta t} \Delta x \Delta y \sum_{i, j}\left|\mathbf{B}_{i, j}^{0}\right|^{2}
$$

where

$$
M=2 \bar{M}+4 \bar{M}^{2}+6\left(\left\|\partial_{x} u^{1}\right\|_{L^{\infty}}+\left\|\partial_{y} u^{2}\right\|_{L^{\infty}}\right)
$$

with

$$
\bar{M}=\max _{i, j}\left\|M_{i, j}\right\| .
$$

Furthermore for the solution of (2.7) we have that

$$
\Delta x \Delta y \sum_{i, j}\left|\mathbf{B}_{i, j}(t)\right|^{2} \leq \mathrm{e}^{M t} \Delta x \Delta y \sum_{i, j}\left|\mathbf{B}_{i, j}^{0}\right|^{2}, \quad t \geq 0
$$

Proof. We shall prove (2.13), the proof of (2.14) is simpler and uses the same basic ideas. Using the notation

$$
\mathbf{B}^{2}=\mathbf{B} \cdot \mathbf{B}=\left(B^{1}\right)^{2}+\left(B^{2}\right)^{2},
$$

and the discrete chain rule, (1.13), we find

$$
\begin{aligned}
2 \mathbf{B}_{i, j}^{n} \cdot D_{t}^{+} \mathbf{B}_{i, j}^{n}= & -\left(\mathbf{u} \cdot D^{\mathrm{upw}}\right)_{i, j}\left(\left(\mathbf{B}_{i, j}^{n}\right)^{2}\right)-\Delta x\left(-\left[u_{i, j}^{1}\right]^{-}\left(D_{x}^{+} \mathbf{B}_{i, j}^{n}\right)^{2}+\left[u_{i, j}^{1}\right]^{+}\left(D_{x}^{-} \mathbf{B}_{i, j}^{n}\right)^{2}\right) \\
& -\Delta y\left(-\left[u_{i, j}^{2}\right]^{-}\left(D_{y}^{+} \mathbf{B}_{i, j}^{n}\right)^{2}+\left[u_{i, j}^{2}\right]^{+}\left(D_{y}^{-} \mathbf{B}_{i, j}^{n}\right)^{2}\right)+2 \mathbf{B}_{i, j}^{n} \cdot M_{i, j} \mathbf{B}_{i, j}^{n}+\mathbf{B}_{i, j}^{n} \cdot D_{\delta}^{2}(\mathbf{u})_{i, j} \mathbf{B}_{i, j}^{n} .
\end{aligned}
$$

For any quantity $Z_{i, j}$, using summation by parts

$$
\begin{aligned}
\sum_{i, j} Z_{i, j} \Delta x \sigma_{\delta}\left(u_{i, j}^{1}\right) D_{x}^{+} D_{x}^{-} Z_{i, j} & =-\Delta x \sum_{i, j} D_{x}^{-}\left(Z_{i, j} \sigma_{\delta}\left(u_{i, j}^{1}\right)\right) D_{x}^{-} Z_{i, j} \\
& =-\Delta x \sum_{i, j} Z_{i, j} D_{x}^{-}\left(\sigma_{\delta}\left(u_{i, j}^{1}\right)\right) D_{x}^{-} Z_{i, j}+\sigma_{\delta}\left(u_{i-1, j}^{1}\right)\left(D_{x}^{-} Z_{i, j}\right)^{2} \\
& \leq-\Delta x \sum_{i, j} \sigma_{\delta}\left(u_{i-1, j}^{1}\right)\left(D_{x}^{-} Z_{i, j}\right)^{2}+2 \sum_{i, j}\left|D_{x}^{-} u_{i, j}^{1}\right|\left(\left|Z_{i, j}\right|+\left|Z_{i-1, j}\right|\right)\left|Z_{i, j}\right| \\
& \leq-\Delta x \sum_{i, j} \sigma_{\delta}\left(u_{i, j}^{1}\right)\left(D_{x}^{+} Z_{i, j}\right)^{2}+4\left\|\partial_{x} u^{1}\right\|_{L^{\infty}\left(\mathbb{R}^{2}\right)} \sum_{i, j}\left(Z_{i, j}\right)^{2} .
\end{aligned}
$$

Since $D_{x}^{+} D_{x}^{-}=D_{x}^{-} D_{x}^{+}$, the term $\left(D_{x}^{+} Z_{i, j}\right)^{2}$ can be replaced by $\left(D_{x}^{-} Z_{i, j}\right)^{2}$ above. Therefore we have that

$$
\sum_{i, j} Z_{i, j} \Delta x \sigma_{\delta}\left(u_{i, j}^{1}\right) D_{x}^{+} D_{x}^{-} Z_{i, j} \leq-\frac{\Delta x}{2} \sum_{i, j} \sigma_{\delta}\left(u_{i, j}^{1}\right)\left(\left(D_{x}^{+} Z_{i, j}\right)^{2}+\left(D_{x}^{-} Z_{i, j}\right)^{2}\right)+4\left\|\partial_{x} u^{1}\right\|_{L^{\infty}\left(\mathbb{R}^{2}\right)} \sum_{i, j}\left(Z_{i, j}\right)^{2}
$$

and similarly

$$
\sum_{i, j} Z_{i, j} \Delta y \sigma_{\delta}\left(u_{i, j}^{2}\right) D_{y}^{+} D_{y}^{-} Z_{i, j} \leq-\frac{\Delta y}{2} \sum_{i, j} \sigma_{\delta}\left(u_{i, j}^{2}\right)\left(\left(D_{y}^{+} Z_{i, j}\right)^{2}+\left(D_{y}^{-} Z_{i, j}\right)^{2}\right)+4\left\|\partial_{y} u^{2}\right\|_{L^{\infty}\left(\mathbb{R}^{2}\right)} \sum_{i, j}\left(Z_{i, j}\right)^{2}
$$


Hence, we get

$$
\begin{aligned}
\sum_{i, j}\left(\mathbf{B}_{i, j}^{n} \cdot D_{\delta}^{2}(\mathbf{u})_{i, j} \mathbf{B}_{i, j}^{n}\right) \leq & -\sum_{i, j} \frac{\Delta x}{2} \sigma_{\delta}\left(u_{i, j}^{1}\right)\left(\left(D_{x}^{+} \mathbf{B}_{i, j}^{n}\right)^{2}+\left(D_{x}^{-} \mathbf{B}_{i, j}^{n}\right)^{2}\right) \frac{\Delta y}{2} \sigma_{\delta}\left(u_{i, j}^{2}\right)\left(\left(D_{y}^{+} \mathbf{B}_{i, j}^{n}\right)^{2}\right. \\
& \left.+\left(D_{y}^{-} \mathbf{B}_{i, j}^{n}\right)^{2}\right)+4\left(\left\|\partial_{x} u^{1}\right\|_{L^{\infty}\left(\mathbb{R}^{2}\right)}+\left\|\partial_{y} u^{2}\right\|_{L^{\infty}\left(\mathbb{R}^{2}\right)}\right) \sum_{i, j}\left(\mathbf{B}_{i, j}^{n}\right)^{2}
\end{aligned}
$$

Note also that by the discrete Leibnitz rule (1.12), we have that for any quantity $Z_{i, j}$,

$$
\sum_{i, j}\left(\mathbf{u} \cdot D^{\mathrm{upw}}\right)_{i, j} Z_{i, j}=-\sum_{i, j} Z_{i, j}\left[D_{x}^{+}\left[u_{i, j}^{1}\right]^{+}+D_{x}^{-}\left[u_{i, j}^{1}\right]^{-}+D_{y}^{+}\left[u_{i, j}^{2}\right]^{+}+D_{y}^{-}\left[u_{i, j}^{2}\right]^{-}\right]
$$

Using this on the first term on the right in (2.15) we find that

$$
\begin{aligned}
\sum_{i, j} 2 \mathbf{B}_{i, j}^{n} D_{t}^{+} \mathbf{B}_{i, j}^{n} \leq & \left(6\left(\left\|\partial_{x} u^{1}\right\|_{L^{\infty}\left(\mathbb{R}^{2}\right)}+\left\|\partial_{y} u^{2}\right\|_{L^{\infty}\left(\mathbb{R}^{2}\right)}\right)+2 \bar{M}\right) \sum_{i, j}\left(\mathbf{B}_{i, j}^{n}\right)^{2}-\sum_{i, j} \Delta x\left(-\left[u_{i, j}^{1}\right]^{-}\left(D_{x}^{+} \mathbf{B}_{i, j}^{n}\right)^{2}\right. \\
& \left.+\left[u_{i, j}^{1}\right]^{+}\left(D_{x}^{-} \mathbf{B}_{i, j}^{n}\right)^{2}\right) \Delta y\left(-\left[u_{i, j}^{2}\right]^{-}\left(D_{y}^{+} \mathbf{B}_{i, j}^{n}\right)^{2}+\left[u_{i, j}^{2}\right]^{+}\left(D_{y}^{-} \mathbf{B}_{i, j}^{n}\right)^{2}\right) \\
& -\sum_{i, j} \frac{\Delta x}{2} \sigma_{\delta}\left(u_{i, j}^{1}\right)\left(\left(D_{x}^{+} \mathbf{B}_{i, j}^{n}\right)^{2}+\left(D_{x}^{-} \mathbf{B}_{i, j}^{n}\right)^{2}\right) \frac{\Delta y}{2} \sigma_{\delta}\left(u_{i, j}^{2}\right)\left(\left(D_{y}^{+} \mathbf{B}_{i, j}^{n}\right)^{2}+\left(D_{y}^{-} \mathbf{B}_{i, j}^{n}\right)^{2}\right)
\end{aligned}
$$

We also have that

$$
D_{t}^{+}\left(\left(\mathbf{B}_{i, j}^{n}\right)^{2}\right)=2 \mathbf{B}_{i, j}^{n} \cdot D_{t}^{+} \mathbf{B}_{i, j}^{n}+\Delta t\left(D_{t}^{+} \mathbf{B}_{i, j}^{n}\right)^{2}
$$

In order to balance the terms, we use the scheme (2.6), and the inequality

$$
\left(\Delta x D_{x}^{+} D_{x}^{-} a_{j}\right)^{2} \leq 2\left(\left(D_{x}^{+} a_{j}\right)^{2}+\left(D_{x}^{-} a_{j}\right)^{2}\right)
$$

Then

$$
\begin{aligned}
\left(D_{t}^{+} \mathbf{B}_{i, j}^{n}\right)^{2} \leq & 2\left(\left(\mathbf{u} \cdot D^{\mathrm{upw}}\right)_{i, j} \mathbf{B}_{i, j}^{n}\right)^{2}+4\left(M_{i, j} \mathbf{B}_{i, j}^{n}\right)^{2}+4\left(D_{\delta}^{2}(\mathbf{u})_{i, j} \mathbf{B}_{i, j}^{n}\right)^{2} \\
\leq & 8\left[\left(\left[u_{i, j}^{1}\right]^{-}\right)^{2}\left(D_{x}^{+} \mathbf{B}_{i, j}^{n}\right)^{2}+\left(\left[u_{i, j}^{1}\right]^{+}\right)^{2}\left(D_{x}^{-} \mathbf{B}_{i, j}^{n}\right)^{2}+\left(\left[u_{i, j}^{2}\right]^{-}\right)^{2}\left(D_{y}^{+} \mathbf{B}_{i, j}^{n}\right)^{2}+\left(\left[u_{i, j}^{2}\right]^{+}\right)^{2}\left(D_{y}^{-} \mathbf{B}_{i, j}^{n}\right)^{2}\right] \\
& +8\left[\left(\Delta x \sigma_{\delta}\left(u_{i, j}^{1}\right) D_{x}^{+} D_{x}^{-} \mathbf{B}_{i, j}^{n}\right)^{2}+\left(\Delta y \sigma_{\delta}\left(u_{i, j}^{2}\right) D_{y}^{+} D_{y}^{-} \mathbf{B}_{i, j}^{n}\right)^{2}\right]+4 \bar{M}^{2}\left(\mathbf{B}_{i, j}^{n}\right)^{2} \\
\leq & 8\left[\left(\left[u_{i, j}^{1}\right]^{-}\right)^{2}\left(D_{x}^{+} \mathbf{B}_{i, j}^{n}\right)^{2}+\left(\left[u_{i, j}^{1}\right]^{+}\right)^{2}\left(D_{x}^{-} \mathbf{B}_{i, j}^{n}\right)^{2}+\left(\left[u_{i, j}^{2}\right]^{-}\right)^{2}\left(D_{y}^{+} \mathbf{B}_{i, j}^{n}\right)^{2}+\left(\left[u_{i, j}^{2}\right]^{+}\right)^{2}\left(D_{y}^{-} \mathbf{B}_{i, j}^{n}\right)^{2}\right] \\
& +16\left[\sigma_{\delta}^{2}\left(u_{i, j}^{1}\right)\left(\left(D_{x}^{+} \mathbf{B}_{i, j}^{n}\right)^{2}+\left(D_{x}^{-} \mathbf{B}_{i, j}^{n}\right)^{2}\right)+\sigma_{\delta}^{2}\left(u_{i, j}^{2}\right)\left(\left(D_{y}^{+} \mathbf{B}_{i, j}^{n}\right)^{2}+\left(D_{y}^{-} \mathbf{B}_{i, j}^{n}\right)^{2}\right)\right] \\
& +4 \bar{M}^{2}\left(\mathbf{B}_{i, j}^{n}\right)^{2} .
\end{aligned}
$$


Using the above and summing over $i$ and $j$,

$$
\begin{aligned}
D_{t}^{+} \sum_{i, j}\left(\mathbf{B}_{i, j}^{n}\right)^{2} \leq & \sum_{i, j}\left[\left|\left[u_{i+1 / 2, j}^{1}\right]^{-}\right|\left(8 \Delta t\left|\left[u_{i+1 / 2, j}^{1}\right]^{-}\right|-\Delta x\right)\left(D_{x}^{+} \mathbf{B}_{i, j}^{n}\right)^{2}\right. \\
& +\left[u_{i-1 / 2, j}^{1}\right]^{+}\left(8 \Delta t\left[u_{i-1 / 2, j}^{1}\right]^{+}-\Delta x\right)\left(D_{x}^{-} \mathbf{B}_{i, j}^{n}\right)^{2} \\
& +\left|\left[u_{i, j+1 / 2}^{2}\right]^{-}\right|\left(8 \Delta t\left|\left[u_{i, j+1 / 2}^{2}\right]^{-}\right|-\Delta y\right)\left(D_{y}^{+} \mathbf{B}_{i, j}^{n}\right)^{2} \\
& +\left[u_{i, j-1 / 2}^{1}\right]^{+}\left(8 \Delta t\left[u_{i, j-1 / 2}^{2}\right]^{+}-\Delta y\right)\left(D_{y}^{-} \mathbf{B}_{i, j}^{n}\right)^{2} \\
& +\sigma_{\delta}\left(u_{i, j}^{1}\right)\left(16 \Delta t \sigma_{\delta}\left(u_{i, j}^{1}\right)-\frac{\Delta x}{2}\right)\left(\left(D_{x}^{+} \mathbf{B}_{i, j}^{n}\right)^{2}+\left(D_{x}^{-} \mathbf{B}_{i, j}^{n}\right)^{2}\right) \\
& \left.+\sigma_{\delta}\left(u_{i, j}^{2}\right)\left(16 \Delta t \sigma_{\delta}\left(u_{i, j}^{2}\right)-\frac{\Delta y}{2}\right)\left(\left(D_{y}^{+} \mathbf{B}_{i, j}^{n}\right)^{2}+\left(D_{y}^{-} \mathbf{B}_{i, j}^{n}\right)^{2}\right)\right] \\
& +\left(2 \bar{M}+4 \bar{M}^{2}+6\left(\left\|\partial_{x} u^{1}\right\|_{L^{\infty}}+\left\|\partial_{y} u^{2}\right\|_{L^{\infty}}\right)\right) \sum_{i, j}\left(\mathbf{B}_{i, j}^{n}\right)^{2} \leq M \sum_{i, j}\left(\mathbf{B}_{i, j}^{n}\right)^{2}
\end{aligned}
$$

if the CFL-condition (2.12) holds. Now (2.13) follows by the discrete Gronwall inequality.

The CFL-condition (2.12) implies that the terms in front of $\left(D_{x, y}^{ \pm} \mathbf{B}_{i, j}^{n}\right)^{2}$ above are all less than $-\Delta x / 2$ or $-\Delta y / 2$. Therefore, as a consequence of the proof, we also get a bound on the spatial variation of $\mathbf{B}_{i, j}^{n}$. Set

$$
\begin{aligned}
\Upsilon_{i, j}^{n}= & \frac{\Delta x}{2}\left(\left(-\left[u_{i, j}^{1}\right]^{-}+\sigma_{\delta}\left(u_{i, j}^{1}\right)\right)\left(D_{x}^{+} \mathbf{B}_{i, j}^{n}\right)^{2}+\left(\left[u_{i, j}^{1}\right]^{+}+\sigma_{\delta}\left(u_{i, j}^{1}\right)\right)\left(D_{x}^{-} \mathbf{B}_{i, j}^{n}\right)^{2}\right) \\
& +\frac{\Delta y}{2}\left(\left(-\left[u_{i, j}^{2}\right]^{-}+\sigma_{\delta}\left(u_{i, j}^{2}\right)\right)\left(D_{y}^{+} \mathbf{B}_{i, j}^{n}\right)^{2}+\left(\left[u_{i, j}^{2}\right]^{+}+\sigma\left(u_{i, j}^{2}\right)\right)\left(D_{y}^{-} \mathbf{B}_{i, j}^{n}\right)^{2}\right) .
\end{aligned}
$$

By (2.15) and (2.13) the following bound holds

$$
\begin{aligned}
\Delta t \sum_{i, j} \Upsilon_{i, j}^{n} & \leq(M+1) \sum_{i, j}\left(\left(\mathbf{B}_{i, j}^{n}\right)^{2}+\left(\mathbf{B}_{i, j}^{n+1}\right)^{2}\right) \\
& \leq 2(M+1) \mathrm{e}^{M(n+1) \Delta t} \sum_{i, j}\left(\mathbf{B}_{i, j}^{0}\right)^{2}
\end{aligned}
$$

Since the scheme uses local upwinding and adds some numerical diffusion, the first part of the updating is monotone in all its arguments. More concretely, set

$$
f\left(Z_{i, j}, Z_{i-1, j}, Z_{i+1, j}, Z_{i, j-1}, Z_{i, j+1}\right)=Z_{i, j}-\Delta t\left(\mathbf{u} \cdot D^{\mathrm{upw}}\right)_{i, j} Z_{i, j}+\Delta t D_{\delta}^{2}(\mathbf{u})_{i, j} Z_{i, j} .
$$

Differentiation and positivity of the coefficients $\sigma_{\delta}$ and the CFL-condition (2.12) show that

$$
\frac{\partial f}{\partial Z_{i, j}} \geq 0, \quad \frac{\partial f}{\partial Z_{i \pm 1, j}} \geq 0 \quad \text { and } \quad \frac{\partial f}{\partial Z_{i, j \pm 1}} \geq 0 .
$$

Next, we prove a bound for the supremum norm, defined as

$$
\left\|\mathbf{B}_{i, j}^{n}\right\|_{L^{\infty}}=\sup _{i, j}\left|B_{i, j}^{1, n}\right|+\sup _{i, j}\left|B_{i, j}^{2, n}\right| .
$$


Lemma 2.2. Let $\mathbf{B}_{i, j}^{n}$ be defined by the scheme (2.8). Then

$$
\left\|\mathbf{B}_{i, j}^{n}\right\|_{L^{\infty}} \leq \mathrm{e}^{C n \Delta t}\left\|\mathbf{B}_{i, j}^{0}\right\|_{L^{\infty}}
$$

where $C=\left\|\partial_{x} u^{1}\right\|_{L^{\infty}}+\left\|\partial_{y} u^{1}\right\|_{L^{\infty}}+\left\|\partial_{x} u^{2}\right\|_{L^{\infty}}+\left\|\partial_{y} u^{2}\right\|_{L^{\infty}}$.

Proof. We write (2.6) as

$$
\begin{aligned}
& B_{i, j}^{1, n+1}=f\left(B_{i, j}^{1, n}, B_{i-1, j}^{1, n}, B_{i+1, j}^{1, n}, B_{i, j-1}^{1, n}, B_{i, j+1}^{1, n}\right)-\Delta t\left(D_{y}^{0} u_{i, j}^{2} B_{i, j}^{1, n}-D_{y}^{0} u_{i, j+1 / 2}^{1} B_{i, j}^{2, n}\right) \\
& B_{i, j}^{2, n+1}=f\left(B_{i, j}^{2, n}, B_{i-1, j}^{2, n}, B_{i+1, j}^{2, n}, B_{i, j-1}^{2, n}, B_{i, j+1}^{2, n}\right)+\Delta t\left(D_{y}^{0} u_{i+1 / 2, j}^{2} B_{i, j}^{1, n}-D_{0}^{y} u_{i+1 / 2, j}^{1} B_{i, j}^{2, n}\right) .
\end{aligned}
$$

Set $\alpha^{n}=\sup _{i, j}\left|B_{i, j}^{1, n}\right|$ and $\beta=\sup _{i, j}\left|B_{i, j}^{2, n}\right|$. Since $f(a, a, a, a, a)=a$ and $f$ is increasing in all its arguments, it follows that

$$
\begin{aligned}
& \alpha^{n+1} \leq \alpha^{n}+\Delta t\left(\left\|\partial_{y} u^{2}\right\|_{L^{\infty}} \alpha^{n}+\left\|\partial_{y} u^{1}\right\|_{L^{\infty}} \beta^{n}\right) \\
& \beta^{n+1} \leq \beta^{n}+\Delta t\left(\left\|\partial_{x} u^{2}\right\|_{L^{\infty}} \alpha^{n}+\left\|\partial_{x} u^{1}\right\|_{L^{\infty}} \beta^{n}\right) .
\end{aligned}
$$

Adding these two inequalities we obtain

$$
\begin{aligned}
\left\|\mathbf{B}_{i, j}^{n+1}\right\|_{L^{\infty}} & \leq\left\|\mathbf{B}_{i, j}^{n}\right\|_{L^{\infty}}+\Delta t\left(\left\|\partial_{x} u^{2}\right\|_{L^{\infty}}+\left\|\partial_{y} u^{2}\right\|_{L^{\infty}}\right) \alpha^{n}+\Delta t\left(\left\|\partial_{x} u^{1}\right\|_{L^{\infty}}+\left\|\partial_{y} u^{1}\right\|_{L^{\infty}}\right) \beta^{n} \\
& \leq(1+C \Delta t)\left\|\mathbf{B}_{i, j}^{n}\right\|_{L^{\infty}}
\end{aligned}
$$

Gronwall's inequality concludes the proof of the lemma.

Remark 2.1. If $\mathbf{u}$ is constant, i.e., $\mathbf{u}=\left(u^{1}, u^{2}\right)$ for two constants $u^{1}$ and $u^{2}$, we can choose $\delta=0$ in the diffusion coefficients $\sigma_{\delta}$. This follows by taking $\delta \leq \min \left\{\left|u_{1}\right|,\left|u_{2}\right|\right\}$ in the case where both $u_{1}, u_{2}$ are away from zero. The case where one or both of the constant velocity fields is zero leads to a single advection equation and we ignore it here. In this special case, the scheme (2.8) reduces to a particularly simple form:

$$
\begin{gathered}
D_{t}^{+} B_{i, j}^{1, n}=-\left[u^{1}\right]^{-} D_{x}^{+}\left(B_{i, j}^{1, n}\right)-\left[u^{1}\right]^{+} D_{x}^{-}\left(B_{i, j}^{1, n}\right)-\left[u^{2}\right]^{-} D_{y}^{+}\left(B_{i, j}^{1, n}\right)-\left[u^{2}\right]^{+} D_{y}^{-}\left(B_{i, j}^{1, n}\right), \\
D_{t}^{+} B_{i, j}^{2, n}=-\left[u^{1}\right]^{-} D_{x}^{+}\left(B_{i, j}^{2, n}\right)-\left[u^{1}\right]^{+} D_{x}^{-}\left(B_{i, j}^{2, n}\right)-\left[u^{2}\right]^{-} D_{y}^{+}\left(B_{i, j}^{2, n}\right)-\left[u^{2}\right]^{+} D_{y}^{-}\left(B_{i, j}^{2, n}\right) .
\end{gathered}
$$

In this case, we have some additional properties. Firstly, we observe that now Lemma 2.1 and Lemma 2.2 give

$$
\Delta x \Delta y \sum_{i, j}\left|\mathbf{B}_{i, j}^{n}\right|^{2} \leq \Delta x \Delta y \sum_{i, j}\left|\mathbf{B}_{i, j}^{0}\right|^{2}, \text { and }\left\|\mathbf{B}_{i, j}^{n}\right\|_{L^{\infty}} \leq\left\|\mathbf{B}_{i, j}^{0}\right\|_{L^{\infty}}
$$

respectively. Also the operator $(\mathbf{u} \cdot D)_{i, j}$ is independent of $i$ and $j$. By Harten's lemma, see [12], and the monotonicity of $f$

where

$$
\left|B_{i, j}^{k, n}\right|_{B . V .} \leq\left|B_{i, j}^{k, 0}\right|_{B . V .}, \text { for } k=1,2,
$$

$$
\left|B_{i, j}^{k, n}\right|_{B . V .}=\Delta y \Delta x \sum_{i, j}\left|D_{x}^{+} B_{i, j}^{k, n}\right|+\left|D_{y}^{+} B_{i, j}^{k, n}\right|
$$

If $\mathbf{u}$ is constant, then we also have that if some discrete divergence of $\mathbf{B}$ initially is zero, then this will remain zero. To see this, let $\mathcal{L} \mathbf{B}_{i, j}^{n}$ be any finite linear combination of $\mathbf{B}_{i, j}^{n}$ for various $i$ 's and $j$ 's, i.e.,

$$
\left(\mathcal{L} \mathbf{B}_{i, j}^{n}\right)=\sum_{m=1}^{2} \sum_{k=1}^{N} \alpha_{k}^{m} B_{i+\sigma(k), j+\kappa(k)}^{m, n},
$$


where $\sigma$ and $\kappa$ are functions taking integer values, and $\alpha_{k}^{m}$ is a constant. Applying $\mathcal{L}$ to the definition of the scheme gives

$$
D_{t}^{+}\left(\mathcal{L} \mathbf{B}_{i, j}^{n}\right)=-(\mathbf{u} \cdot D)\left(\mathcal{L} \mathbf{B}_{i, j}^{n}\right) .
$$

In particular, if $\mathcal{L} \mathbf{B}_{i, j}^{0}=0$ then $\mathcal{L} \mathbf{B}_{i, j}^{n}=0$ for $n>0$. Since any discrete divergence is of the same type as $\mathcal{L}$, any zero initial discrete divergence will remain zero. We also remark that for constant velocity, the CFL-condition can be relaxed to

$$
\Delta t \max \left\{\frac{\left|u^{1}\right|}{\Delta x}, \frac{\left|u^{2}\right|}{\Delta y}\right\} \leq \frac{1}{2} .
$$

Hence, in the case of constant velocity fields, the scheme (2.8) is non-oscillatory and preserves any discrete divergence operator. These are important structural properties of the scheme and explain some of the results in the section on numerical experiments.

\subsection{Convergence}

It follows directly from the $L^{2}$ bound in Lemma 2.1 that that the sequence $\left\{\mathbf{B}^{\Delta t}\right\}$ is weakly compact in $L^{2}\left(\mathbb{R}^{2} \times[0, T]\right)$. By the $L^{2}$ bound we infer the existence of a function $\mathbf{B}(x, y, t)$ such that

$$
\mathbf{B}^{\Delta t} \rightarrow \mathbf{B}, \quad \text { in } L^{2}\left(\mathbb{R}^{2} \times[0, T]\right) \text { as } \Delta t \rightarrow 0 .
$$

Theorem 2.1. Let $\mathbf{B}^{n}$ be defined by the scheme (2.8), and assume that $\left\|\mathbf{B}_{0}\right\|_{L^{2}\left(\mathbb{R}^{2}\right)}$ is finite. If $\mathbf{u} \in C^{2}\left(\mathbb{R}^{2}\right)$ with bounded derivatives, and $\Delta t$ satisfies the CFL-condition (2.12), then there exists a subsequence $\left\{\Delta t_{j}\right\}$ such that $\mathbf{B}^{\Delta t_{j}} \rightarrow \mathbf{B}$ in $L^{2}\left(\mathbb{R}^{2} \times[0, T]\right)$. Furthermore, the limit $\mathbf{B}=\left(B^{1}, B^{2}\right)$ is a weak solution of (1.6), with the property that

$$
\|\mathbf{B}(\cdot, T)\|_{L^{2}\left(\mathbb{R}^{2}\right)} \leq C_{T}\left\|\mathbf{B}_{0}\right\|_{L^{2}\left(\mathbb{R}^{2}\right)}, \quad T \geq 0,
$$

where $C_{T}$ is a finite constant depending on $\mathbf{u}$ and $T$. The same results hold for the approximations generated by the semi-discrete scheme (2.7).

Proof. We must show that $\mathbf{B}$ is a weak solution, and we shall do this for the first component $B^{1}$. Identical arguments apply to $B^{2}$. To demonstrate that $\mathbf{B}$ is a weak solution of (1.6) we must then show that $B^{1}$ satisfies

$$
\begin{aligned}
\mathcal{W}_{\varphi}(\mathbf{B}):=\iiint_{\mathbb{R}^{2} \times[0, \infty)} B^{1} \partial_{t} \varphi+u^{1} B^{1} \partial_{x} \varphi+u^{2} B^{1} \partial_{y} \varphi-B^{1} \partial_{x}\left(u^{1}\right) \varphi-B^{2} \partial_{y}\left(u^{1}\right) \varphi \mathrm{d} x \mathrm{~d} y \mathrm{~d} t \\
\quad+\iint_{\mathbb{R}^{2}} B_{0}^{1} \varphi(x, y, 0) \mathrm{d} x \mathrm{~d} y=0,
\end{aligned}
$$

for all test functions $\varphi \in C_{0}^{\infty}(\Pi)$, where $\Pi=\mathbb{R}^{2} \times[0, \infty)$.

Choose a test function $\varphi$ and set

$$
\varphi_{i, j}^{n}=\frac{1}{\Delta t \Delta x \Delta y} \iiint_{I_{i, j}^{n}} \varphi(x, y, t) \mathrm{d} x \mathrm{~d} y \mathrm{~d} t .
$$

In order to prove convergence to a weak solution (2.21), we need to work with the "conservative form" of the scheme (2.10).

To save space, set $\Delta=\Delta t \Delta x \Delta y$, multiply (2.10) by $\Delta \varphi_{i, j}^{n}$, sum over $n=0, \ldots, \infty$, and $(i, j)$ in $\mathbb{Z}^{2}$, and sum by parts to arrive at

$$
\alpha_{1}+\alpha_{2}+\alpha_{3}+\alpha_{4}+\alpha_{5}+\alpha_{6}+\alpha_{7}+\alpha_{8}+\alpha_{9}+\alpha_{10}+\alpha_{11}+\alpha_{12}=0
$$


where

$$
\begin{aligned}
\alpha_{1} & =\Delta \sum_{n=1}^{\infty} \sum_{i, j} B_{i, j}^{1, n} D_{t}^{-} \varphi_{i, j}^{n}, & \alpha_{2} & =\Delta x \Delta y \sum_{i, j} B_{i, j}^{1,0} \varphi_{i, j}^{0}, \\
\alpha_{3} & =\Delta \sum_{n=1}^{\infty} \sum_{i, j} u_{i, j}^{1} B_{i, j}^{1}\left(D_{x}^{0} \varphi_{i, j}^{n}\right), & \alpha_{4} & =\Delta \sum_{n=1}^{\infty} \sum_{i, j} u_{i, j}^{2} B_{i, j}^{1}\left(D_{y}^{0} \varphi_{i, j}^{n}\right), \\
\alpha_{5} & =-\Delta \sum_{n=1}^{\infty} \sum_{i, j} \varphi_{i, j}^{n} B_{i, j}^{1}\left(D_{x}^{0} u_{i, j}^{1}\right), & \alpha_{6} & =\Delta \sum_{n=1}^{\infty} \sum_{i, j} \varphi_{i, j}^{n} B_{i, j}^{2}\left(D_{y}^{0} u_{i, j}^{1}\right),
\end{aligned}
$$

and

$$
\begin{aligned}
\alpha_{7} & =-\Delta x \Delta \sum_{n=1}^{\infty} \sum_{i, j} D_{x}^{+} D_{x}^{-}\left(\left|u_{i, j}^{1}\right|_{\delta} \varphi_{i, j}^{n}\right) B_{i, j}^{1, n}, \\
\alpha_{8} & =-\Delta y \Delta \sum_{n=1}^{\infty} \sum_{i, j} D_{y}^{+} D_{y}^{-}\left(\left|u_{i, j}^{2}\right|_{\delta} \varphi_{i, j}^{n}\right) B_{i, j}^{1, n}, \\
\alpha_{9} & =(\Delta x)^{2} \Delta \sum_{n=1}^{\infty} \sum_{i, j} D_{x}^{+}\left(\varphi_{i, j}^{n} D_{x}^{-}\left(u_{i, j}^{1}\right)\right) D_{x}^{+}\left(B_{i, j}^{1, n}\right), \\
\alpha_{10} & =(\Delta y)^{2} \Delta \sum_{n=1}^{\infty} \sum_{i, j} D_{y}^{+}\left(\varphi_{i, j}^{n} D_{y}^{-}\left(u_{i, j}^{2}\right)\right) D_{y}^{+}\left(B_{i, j}^{1, n}\right), \\
\alpha_{11} & =-(\Delta x)^{2} \Delta \sum_{n=1}^{\infty} \sum_{i, j} \varphi_{i, j}^{n} D_{x}^{+} D_{x}^{-}\left(u_{i, j}^{1}\right) D_{x}^{+}\left(B_{i, j}^{1, n}\right), \\
\alpha_{12} & =(\Delta y)^{2} \Delta \sum_{n=1}^{\infty} \sum_{i, j} \varphi_{i, j}^{n} D_{y}^{+} D_{y}^{-}\left(u_{i, j}^{2}\right) D_{y}^{+}\left(B_{i, j}^{1, n}\right) .
\end{aligned}
$$

We claim that since $\mathbf{B}^{\Delta t} \in L^{2}\left(\mathbb{R}^{2} \times[0, T]\right)$ for all finite $T$, all the terms $\alpha_{7}, \alpha_{8}, \ldots, \alpha_{12}$ vanish a $\Delta \rightarrow 0$. We can estimate $\alpha_{7}$ and $\alpha_{8}$ as follows:

$$
\begin{aligned}
\alpha_{7}^{2} & \leq \Delta x^{2}\left(\Delta \sum_{n, i, j}\left(D_{x}^{+} D_{x}^{-}\left(\left|u_{i, j}^{1}\right|_{\delta} \varphi_{i, j}^{n}\right)\right)^{2}\right)\left(\Delta \sum_{n, i, j}\left(B_{i, j}^{1, n}\right)^{2}\right) \\
& \leq C \Delta x^{2} \quad \rightarrow 0, \quad \text { as } \quad \Delta x \rightarrow 0,
\end{aligned}
$$

for some constant $C$ which is independent of $\Delta t$. When we estimate $\alpha_{9}, \ldots, \alpha_{12}$ we have an extra $\Delta x$ or $\Delta y$ which can be used to "remove the discrete derivative" from $\mathbf{B}$. This is done for $\alpha_{9}$ as

$$
\begin{aligned}
\alpha_{9} & \leq \Delta x \sum_{n, i, j}\left|D_{x}^{+}\left(\varphi_{i, j}^{n} D_{x}^{-} u_{i, j}^{1}\right)\right|\left(\left|B_{i+1, j}^{1, n}\right|+\left|B_{i, j}^{1, n}\right|\right) \\
& \leq \Delta x\left(\sum_{n, i, j}\left(D_{x}^{+}\left(\varphi_{i, j}^{n} D_{x}^{-} u_{i, j}^{1}\right)\right)^{2}\right)^{1 / 2}\left(4 \sum_{n, i, j}\left(B_{i, j}^{1, n}\right)^{2}\right)^{1 / 2} \\
& \leq C \Delta x
\end{aligned}
$$

for some constant $C$ which is independent of $\Delta t$. 
Using that $\mathbf{B}^{\Delta t} \rightarrow \mathbf{B}$ as $\Delta t \rightarrow 0$ and that $\mathbf{u}$ and $\varphi$ are smooth enough, we can use standard arguments to show that as $\Delta t, \Delta x, \Delta y \rightarrow 0$,

$$
\begin{aligned}
\alpha_{1} & \rightarrow \iiint_{\Pi} B^{1} \partial_{t} \varphi \mathrm{d} x \mathrm{~d} y \mathrm{~d} t, & \alpha_{2} & \rightarrow \iint_{\mathbb{R}^{2}} B_{0}^{1} \varphi(x, y, 0) \mathrm{d} x \mathrm{~d} y \\
\alpha_{3} & \rightarrow \iiint_{\Pi} u^{1} B^{1} \partial_{x} \varphi \mathrm{d} x \mathrm{~d} y \mathrm{~d} t, & \alpha_{4} & \rightarrow \iiint_{\Pi} u^{2} B^{1} \partial_{y} \varphi \mathrm{d} x \mathrm{~d} y \mathrm{~d} t \\
\alpha_{5} & \rightarrow \iiint_{\Pi} \partial_{x}\left(u^{1}\right) B^{1} \varphi \mathrm{d} x \mathrm{~d} y \mathrm{~d} t, & \alpha_{6} & \rightarrow \iint_{\Pi} \partial_{y}\left(u^{1}\right) B^{1} \varphi \mathrm{d} x \mathrm{~d} y \mathrm{~d} t .
\end{aligned}
$$

Hence, we have shown that the approximations defined by (2.10) and (2.11) converge to a weak solution of (1.6).

\subsection{Divergence bounds}

In this section, we show that we can bound the divergence of the semi-discrete scheme (2.7) under certain assumptions.

In order to motivate some rather long calculations, we start by considering the exact equations

$$
\begin{aligned}
& B_{t}^{1}+u^{1} B_{x}^{1}+u^{2} B_{y}^{1}=-u_{y}^{2} B^{1}+u_{y}^{1} B^{2} \\
& B_{t}^{2}+u^{1} B_{x}^{2}+u^{2} B_{y}^{2}=u_{x}^{2} B^{1}-u_{x}^{1} B^{2} .
\end{aligned}
$$

Setting $d=B_{x}^{1}+B_{y}^{2}$, differentiating the first equation with respect to $x$ and the second with respect to $y$ and adding yields

$$
d_{t}+u^{1} d_{x}+u^{2} d_{y}=-\left(u_{x}^{1}+u_{y}^{2}\right) d .
$$

Multiplying by $2 d$, and using Leibnitz' rule (again) yields

$$
d_{t}^{2}+\left(u^{1} d^{2}\right)_{x}+\left(u^{2} d^{2}\right)_{y}=-\left(u_{x}^{1}+u_{y}^{2}\right) d^{2} .
$$

Integrating over $x$ and $y$ gives

$$
\frac{\mathrm{d}}{\mathrm{d} t}\|d(\cdot, t)\|_{L^{2}\left(\mathbb{R}^{2}\right)}^{2} \leq\|\operatorname{div} \mathbf{u}\|_{L^{\infty}\left(\mathbb{R}^{2}\right)}\|d(\cdot, t)\|_{L^{2}\left(\mathbb{R}^{2}\right)}^{2} .
$$

We wish to "replicate" this calculation for the approximations generated by the semi-discrete scheme. There are three obstacles in the way of doing so, firstly the approximations do not satisfy (2.22) and (2.23), but (2.7). Secondly we used Leibnitz' rule to arrive at (2.24), this rule do not hold exactly for the discrete differentiation operators $D_{x}^{0}$ and $D_{y}^{0}$. Thirdly we used the chain rule to arrive at (2.24), this is not exact for discrete derivatives.

We start by considering the scheme in the form (2.9), which reads

$$
\begin{aligned}
\frac{\mathrm{d}}{\mathrm{d} t} B_{i, j}^{1}= & -u_{i, j}^{1} D_{x}^{0} B_{i, j}^{1}-u_{i, j}^{2} D_{y}^{0} B_{i, j}^{1}-\left(D_{y}^{0} u_{i, j}^{2}\right) B_{i, j}^{1}+\left(D_{y}^{0} u_{i, j}^{1}\right) B_{i, j}^{2} \\
& +\frac{\Delta x}{2}\left|u_{i, j}^{1}\right|_{\delta} D_{x}^{+} D_{x}^{-} B_{i, j}^{1}+\frac{\Delta y}{2}\left|u_{i, j}^{2}\right|_{\delta} D_{y}^{+} D_{y}^{-} B_{i, j}^{1} \\
\frac{\mathrm{d}}{\mathrm{d} t} B_{i, j}^{2}= & -u_{i, j}^{1} D_{x}^{0} B_{i, j}^{2}-u_{i, j}^{2} D_{y}^{0} B_{i, j}^{2}+\left(D_{x}^{0} u_{i, j}^{2}\right) B_{i, j}^{1}-\left(D_{x}^{0} u_{i, j}^{1}\right) B_{i, j}^{2} \\
& +\frac{\Delta x}{2}\left|u_{i, j}^{1}\right|_{\delta} D_{x}^{+} D_{x}^{-} B_{i, j}^{2}+\frac{\Delta y}{2}\left|u_{i, j}^{2}\right|_{\delta} D_{y}^{+} D_{y}^{-} B_{i, j}^{2} .
\end{aligned}
$$


In order to replace the exact Leibnitz rule, for a smooth function $a(x)$, and a sequence $\left\{b_{i}\right\}$, we have

$$
\begin{aligned}
D^{0}\left(a_{i} b_{i}\right) & =b_{i} D^{0}\left(a_{i}\right)+\frac{1}{2}\left(a_{i+1} D^{+} b_{i}+a_{i-1} D^{-} b_{i}\right) \\
= & b_{i} D^{0}\left(a_{i}\right)+\frac{1}{2}\left[\left(a_{i}+a_{i}^{\prime} \Delta x+\frac{\Delta x^{2}}{2} a^{\prime \prime}\left(\xi_{i+1 / 2}\right)\right) D^{+} b_{i}+\left(a_{i}-a_{i}^{\prime} \Delta x+\frac{\Delta x^{2}}{2} a^{\prime \prime}\left(\xi_{i-1 / 2}\right)\right) D^{-} b_{i}\right] \\
= & a_{i} D^{0} b_{i}+b_{i} D^{0} a_{i} \leftarrow \text { "Leibnitz part" } \\
& +\underbrace{\frac{\Delta x^{2}}{2} a_{i}^{\prime} D^{+} D^{-} b_{i}+\frac{\Delta x^{2}}{4}\left(a^{\prime \prime}\left(\xi_{i+1 / 2}\right) D^{+} b_{i}+a^{\prime \prime}\left(\xi_{i-1 / 2}\right) D^{-} b_{i}\right)}_{\text {"discrete correction" }}
\end{aligned}
$$

where we use the notation $a_{i}=a\left(x_{i}\right), a_{i}^{\prime}=a^{\prime}\left(x_{i}\right)$ and $\xi_{i \pm 1 / 2}$ is between $x_{i}$ and $x_{i \pm 1}$.

We shall first apply $D_{x}^{0}$ to $(2.27), D_{y}^{0}$ to to $(2.28)$ and add the results. The "Leibnitz" part of this will give the discrete equivalent of (2.26), with central differences replacing derivatives, and $d$ replaced by

$$
d_{i, j}=D_{x}^{0} B_{i, j}^{1}+D_{y}^{0} B_{i, j}^{2}
$$

Concretely we get

$$
\begin{array}{r}
\frac{\mathrm{d}}{\mathrm{d} t} d_{i, j}=- \\
u_{i, j}^{1} D_{x}^{0} d_{i, j}-u_{i, j}^{2} D_{y}^{0} d_{i, j}-\left(D_{x}^{0} u_{i, j}^{1}+D_{y}^{0} u_{i, j}^{2}\right) d_{i, j} \\
+ \text { discrete correction terms }+ \text { terms from } D^{+} D^{-} .
\end{array}
$$

Next, we shall multiply this with $d_{i, j}$, and use the chain rule (1.13). In order to get a useful form of the numerical diffusion, we must convert (2.29) to upwind form. The formula for doing so reads

$$
a_{i} D^{0} b_{i}=a_{i}^{+} D^{-} b_{i}+a_{i}^{-} D^{+} b_{i}-\Delta x\left|a_{i}\right| D^{+} D^{-} b_{i} .
$$

Consequently, the upwind form of the equation for the discrete divergence is

$$
\begin{aligned}
\frac{\mathrm{d}}{\mathrm{d} t} d_{i, j}=- & {\left[u_{i, j}^{1}\right]^{-} D_{x}^{+} d_{i, j}-\left[u_{i, j}^{1}\right]^{+} D_{x}^{-} d_{i, j}-\left[u_{i, j}^{2}\right]^{-} D_{y}^{+} d_{i, j}-\left[u_{i, j}^{2}\right]^{+} D_{y}^{-} d_{i, j} } \\
& -\left(D_{x}^{0} u_{i, j}^{1}+D_{y}^{0} u_{i, j}^{2}\right) d_{i, j}+\underbrace{\Delta x\left|u_{i, j}^{1}\right| D_{x}^{+} D_{x}^{-} d_{i, j}+\Delta y\left|u_{i, j}^{2}\right| D_{y}^{+} D_{y}^{-} d_{i, j}}_{\text {"upwind diffusion" }} \\
& + \text { discrete correction terms + terms from } D^{+} D^{-} .
\end{aligned}
$$

Now we multiply this with $2 d_{i, j}$, and use the discrete chain rule (1.13) to get

$$
\begin{aligned}
\frac{\mathrm{d}}{\mathrm{d} t} d_{i, j}^{2}= & -\left[u_{i, j}^{1}\right]^{-} D_{x}^{+} d_{i, j}^{2}-\left[u_{i, j}^{1}\right]^{+} D_{x}^{-} d_{i, j}^{2}-\left[u_{i, j}^{2}\right]^{-} D_{y}^{+} d_{i, j}^{2}-\left[u_{i, j}^{2}\right]^{+} D_{y}^{-} d_{i, j}^{2}-\left(D_{x}^{0} u_{i, j}^{1}+D_{y}^{0} u_{i, j}^{2}\right) d_{i, j}^{2} \\
& +\Delta x\left(\left[u_{i, j}^{1}\right]^{-}\left(D_{x}^{+} d_{i, j}\right)^{2}-\left[u_{i, j}^{1}\right]^{+}\left(D_{x}^{-} d_{i, j}\right)^{2}\right) \\
& \left.+\Delta y\left(\left[u_{i, j}^{2}\right]^{-}\left(D_{y}^{+} d_{i, j}\right)^{2}-\left[u_{i, j}^{2}\right]^{+}\left(D_{y}^{-} d_{i, j}\right)^{2}\right)\right\} \leftarrow \text { signed terms } \\
& \left.+2 d_{i, j} \text { (upwind diffusion }+ \text { discrete correction }+ \text { terms from } D^{+} D^{-}\right) .
\end{aligned}
$$


The next step is summing the above equation over $i$ and $j$, using partial summation and other tricks to bound the right hand side. The first four terms on the right hand side of (2.31) are bounded as follows

$$
\begin{aligned}
\left|\sum_{i, j}\left[u_{i, j}^{1}\right]^{-} D_{x}^{+} d_{i, j}^{2}\right| & =\left|\sum_{i, j} D_{x}^{-}\left(\left[u_{i, j}^{1}\right]^{-}\right) d_{i, j}^{2}\right| \\
& \leq\left\|\partial_{x} u^{1}\right\|_{L^{\infty}\left(\mathbb{R}^{2}\right)} \sum_{i, j} d_{i, j}^{2} .
\end{aligned}
$$

The fifth term on the right side of (2.31) has the same type of bound. Therefore

$$
\begin{aligned}
\frac{\mathrm{d}}{\mathrm{d} t} d_{i, j}^{2} \leq & 3\left(\left\|\partial_{x} u^{1}\right\|_{L^{\infty}\left(\mathbb{R}^{2}\right)}+\left\|\partial_{y} u^{2}\right\|_{L^{\infty}\left(\mathbb{R}^{2}\right)}\right) \sum_{i, j} d_{i, j}^{2}+\sum_{i, j} \text { signed terms } \\
& \left.+2 d_{i, j} \text { (upwind diffusion }+ \text { discrete correction }+ \text { terms from } D^{+} D^{-}\right) .
\end{aligned}
$$

Next, let us tackle the "discrete correction" terms. These are terms coming from applying $D^{0}$ to the first order differences in (2.27) and (2.28). Furthermore, these are essentially of two types; a correction for $D_{x}^{0}$ applied to $u_{i, j}^{1} D_{x}^{0} B_{i, j}^{1}$, and a correction for $D_{x}^{0}$ applied to $\left(D_{x}^{0} u_{i, j}^{2}\right) B_{i, j}^{1}$. Now the correction part of $D_{x}^{0}\left(u_{i, j}^{1} D_{x}^{0} B_{i, j}^{1}\right)$ equals

$$
\frac{\Delta x^{2}}{2} u_{i, j}^{1,{ }^{\prime}} D_{x}^{+} D_{x}^{-} D_{x}^{0} B_{i, j}^{1}+\frac{\Delta x^{2}}{4}\left(u_{i+1 / 2, j}^{1, \prime \prime} D_{x}^{+} D_{x}^{0} B_{i, j}^{1}+u_{i-1 / 2, j}^{1, \prime \prime} D_{x}^{-} D_{x}^{0} B_{i, j}^{1}\right) .
$$

We must multiply this by $2 d_{i, j}$ and sum over $i$ and $j$ and bound the result. For the second term of this part of the correction we have

$$
\begin{aligned}
\left|\frac{1}{4} \sum_{i, j} u_{i+1 / 2, j}^{1, \prime \prime} 2 d_{i, j} \Delta x^{2} D_{x}^{+} D_{x}^{0} B_{i, j}^{1}\right| & \leq \frac{1}{2}\left\|\partial_{x}^{2} u^{1}\right\|_{L^{\infty}\left(\mathbb{R}^{2}\right)} \sum_{i, j}\left|d_{i, j}\right| \frac{1}{2}\left(\left|B_{i+2, j}^{1}\right|+\left|B_{i+1, j}^{1}\right|+\left|B_{i, j}^{1}\right|+\left|B_{i-1, j}^{1}\right|\right) \\
& \leq \frac{1}{2}\left\|\partial_{x}^{2} u^{1}\right\|_{L^{\infty}\left(\mathbb{R}^{2}\right)}\left(\sum_{i, j} d_{i, j}^{2}+\sum_{i, j}\left(B_{i, j}^{1}\right)^{2}\right) .
\end{aligned}
$$

The sum coming from the third term in (2.32) has the same bound. To bound the sum coming from the first term in (2.32) we use summation by parts

$$
\begin{aligned}
\left|\frac{1}{2} \sum_{i, j} 2 d_{i, j} u_{i, j}^{1, \prime} \Delta x^{2} D_{x}^{+} D_{x}^{-} D_{x}^{0} B_{i, j}^{1}\right|= & \frac{1}{2}\left|\sum_{i, j} D_{x}^{-}\left(u_{i, j}^{1,{ }^{\prime}} d_{i, j}\right) \Delta x^{2} D_{x}^{-} D_{x}^{0} B_{i, j}^{1}\right| \\
= & \frac{1}{2}\left|\sum_{i, j}\left(u_{i, j}^{1,{ }^{\prime}} D_{x}^{-} d_{i, j}+d_{i-1, j} D_{x}^{-} u_{i, j}^{1,}\right) \Delta x^{2} D_{x}^{-} D_{x}^{0} B_{i, j}^{1}\right| \\
\leq & \left\|\partial_{x}^{2} u^{1}\right\|_{L^{\infty}\left(\mathbb{R}^{2}\right)} \sum_{i, j} d_{i, j}^{2}+\left(B_{i, j}^{1}\right)^{2} \\
& +\frac{1}{2}\left\|\partial_{x} u^{1}\right\|_{L^{\infty}\left(\mathbb{R}^{2}\right)} \sum_{i, j} \frac{\Delta x}{4 \varepsilon}\left(D_{x}^{-} B_{i, j}^{1}\right)^{2}+\Delta x \varepsilon\left(D_{x}^{-} d_{i, j}\right)^{2}
\end{aligned}
$$

where $\varepsilon$ is a positive number yet to be determined. The last term here looks threatening, but we shall look for countermeasures. Next the correction part of $D_{x}^{0}\left(\left(D_{x}^{0} u_{i, j}^{2}\right) B_{i, j}^{1}\right)$ is

$$
c_{i, j}:=\frac{\Delta x^{2}}{2} D_{x}^{0}\left(u_{i, j}^{2, \prime}\right) D_{x}^{+} D_{x}^{-} B_{i, j}^{1}+\frac{\Delta x^{2}}{4}\left(D_{x}^{0}\left(u_{i+1 / 2, j}^{2, \prime \prime}\right) D_{x}^{+} B_{i, j}^{1}+D_{x}^{0}\left(u_{i-1 / 2, j}^{2, \prime \prime}\right) D_{x}^{-} B_{i, j}^{1}\right) .
$$


Now we can use $\Delta x^{2}$ to remove both discrete derivatives of $B_{i, j}^{1}$ in the first term, and one from $B_{i, j}^{1}$ and one from $u_{i, j}^{2,{ }^{\prime \prime}}$ in the second term, and conclude that

$$
\sum_{i, j} c_{i, j} 2 d_{i, j} \leq C\left\|\partial_{x}^{2} u^{2}\right\|_{L^{\infty}\left(\mathbb{R}^{2}\right)} \sum_{i, j} d_{i, j}^{2}+\left(B_{i, j}^{1}\right)^{2}
$$

where $C$ equals 8 , and is in any case independent of $\Delta x$.

The rest of the discrete correction terms are similar to those we have bounded, and we end up with the bound

$$
\begin{aligned}
& \sum_{i, j} 2 d_{i, j}(\text { discrete correction }) \leq C\left(\left\|\partial_{x}^{2} u^{1}\right\|_{L^{\infty}\left(\mathbb{R}^{2}\right)}+\left\|\partial_{y}^{2} u^{1}\right\|_{L^{\infty}\left(\mathbb{R}^{2}\right)}+\left\|\partial_{x}^{2} u^{2}\right\|_{L^{\infty}\left(\mathbb{R}^{2}\right)}+\left\|\partial_{y}^{2} u^{2}\right\|_{L^{\infty}\left(\mathbb{R}^{2}\right)}\right) \\
& \quad \times \sum_{i, j}\left(d_{i, j}^{2}+\mathbf{B}_{i, j}^{2}\right)+C\left(\left\|\partial_{x} u^{1}\right\|_{L^{\infty}\left(\mathbb{R}^{2}\right)}+\left\|\partial_{y} u^{1}\right\|_{L^{\infty}\left(\mathbb{R}^{2}\right)}+\left\|\partial_{x} u^{2}\right\|_{L^{\infty}\left(\mathbb{R}^{2}\right)}+\left\|\partial_{y} u^{2}\right\|_{L^{\infty}\left(\mathbb{R}^{2}\right)}\right) \\
& \quad \times \sum_{i, j} \frac{1}{\varepsilon}\left(\Delta x\left(D_{x}^{-} B_{i, j}^{1}\right)^{2}+\Delta y\left(D_{y}^{-} B_{i, j}^{2}\right)^{2}\right)+\varepsilon\left(\Delta x\left(D_{x}^{-} d_{i, j}\right)^{2}+\Delta y\left(D_{y}^{-} d_{i, j}\right)^{2}\right)
\end{aligned}
$$

for some finite constant $C$ which does not depend on $\Delta x$ or $\Delta y$.

Now we bound the terms coming from the "upwind diffusion", here we must bound terms like

$$
\begin{aligned}
\sum_{i, j} 2 d_{i, j} \Delta x\left|u_{i, j}^{1}\right| D_{x}^{+} D_{x}^{-} d_{i, j} & \leq-2 \Delta x \sum_{i, j} D_{x}^{-}\left(\left|u_{i, j}^{1}\right| d_{i, j}\right) D_{x}^{-} d_{i, j} \\
& =-2 \Delta x \sum_{i, j}\left|u_{i, j}^{1}\right|\left(D_{x}^{-} d_{i, j}\right)^{2}-d_{i-1, j} D_{x}^{-}\left|u_{i, j}^{1}\right| D_{x}^{-} d_{i, j} \\
& \leq-2 \Delta x \sum_{i, j}\left|u_{i, j}^{1}\right|\left(D_{x}^{-} d_{i, j}\right)^{2}+10\left\|\partial_{x} u^{1}\right\|_{L^{\infty}\left(\mathbb{R}^{2}\right)} \sum_{i, j} d_{i, j}^{2} .
\end{aligned}
$$

Then we have that

$$
\begin{aligned}
\sum_{i, j} 2 d_{i, j} \text { (upwind diffusion) } \leq & -\sum_{i, j} \Delta x\left|u_{i, j}^{1}\right|\left(D_{x}^{-} d_{i, j}\right)^{2}+\Delta y\left|u_{i, j}^{2}\right|\left(D_{y}^{-} d_{i, j}\right)^{2} \\
& +10\left(\left\|\partial_{x} u^{1}\right\|_{L^{\infty}\left(\mathbb{R}^{2}\right)}+\left\|\partial_{y} u^{2}\right\|_{L^{\infty}\left(\mathbb{R}^{2}\right)}\right) \sum_{i, j} d_{i, j}^{2}
\end{aligned}
$$

Then we turn to the "terms from $D^{+} D^{-}$". These are the result of applying $D_{x}^{0}$ to the second order differences in $(2.27), D_{y}^{0}$ to the second order differences in (2.28) and adding the results. We have that

$$
\begin{aligned}
D_{x}^{0}\left(\frac{\Delta x}{2}\left|u_{i, j}^{1}\right|_{\delta} D_{x}^{+} D_{x}^{-} B_{i, j}^{1}+\frac{\Delta y}{2}\left|u_{i, j}^{2}\right|_{\delta} D_{y}^{+} D_{y}^{-} B_{i, j}^{1}\right) \\
=\frac{\Delta x}{2}\left[\left|u_{i, j}^{1}\right|_{\delta} D_{x}^{+} D_{x}^{-} D_{x}^{0}\left(B_{i, j}^{1}\right)+\frac{1}{2}\left(D_{x}^{-} D_{x}^{+} B_{i+1, j}^{1} D_{x}^{+}\left|u_{i, j}^{1}\right|_{\delta}+D_{x}^{+} D_{x}^{-} B_{i-1, j}^{1} D_{x}^{-}\left|u_{i, j}^{1}\right|_{\delta}\right)\right] \\
\quad+\frac{\Delta y}{2}\left[\left|u_{i, j}^{2}\right|_{\delta} D_{y}^{+} D_{y}^{-} D_{x}^{0}\left(B_{i, j}^{1}\right)+\frac{1}{2}\left(D_{y}^{-} D_{y}^{+} B_{i+1, j}^{1} D_{x}^{+}\left|u_{i, j}^{2}\right|_{\delta}+D_{y}^{+} D_{y}^{-} B_{i-1, j}^{1} D_{x}^{-}\left|u_{i, j}^{2}\right|_{\delta}\right)\right]
\end{aligned}
$$


and

$$
\begin{aligned}
D_{y}^{0}\left(\frac{\Delta x}{2}\left|u_{i, j}^{1}\right|_{\delta} D_{x}^{+} D_{x}^{-} B_{i, j}^{2}+\frac{\Delta y}{2}\left|u_{i, j}^{2}\right|_{\delta} D_{y}^{+} D_{y}^{-} B_{i, j}^{2}\right)=\frac{\Delta x}{2}\left[\left|u_{i, j}^{1}\right|_{\delta} D_{x}^{+} D_{x}^{-} D_{y}^{0}\left(B_{i, j}^{2}\right)\right. \\
\left.+\frac{1}{2}\left(D_{x}^{-} D_{x}^{+} B_{i, j+1}^{2} D_{y}^{+}\left|u_{i, j}^{1}\right|_{\delta}+D_{x}^{+} D_{x}^{-} B_{i, j-1}^{2} D_{y}^{-}\left|u_{i, j}^{1}\right|_{\delta}\right)\right] \\
+\frac{\Delta y}{2}\left[\left|u_{i, j}^{2}\right|_{\delta} D_{y}^{+} D_{y}^{-} D_{y}^{0}\left(B_{i, j}^{2}\right)+\frac{1}{2}\left(D_{y}^{-} D_{y}^{+} B_{i, j+1}^{2} D_{y}^{+}\left|u_{i, j}^{2}\right|_{\delta}+D_{y}^{+} D_{y}^{-} B_{i, j-1}^{2} D_{y}^{-}\left|u_{i, j}^{2}\right|_{\delta}\right)\right] .
\end{aligned}
$$

Adding these we get

$$
\begin{aligned}
\text { "terms from } D^{+} D^{-"}= & \frac{\Delta x}{2}\left|u_{i, j}^{1}\right|_{\delta} D_{x}^{+} D_{x}^{-} d_{i, j}+\frac{\Delta y}{2}\left|u_{i, j}^{2}\right|_{\delta} D_{y}^{+} D_{y}^{-} d_{i, j} \\
& + \text { second order corrections. }
\end{aligned}
$$

Multiplied by $2 d_{i, j}$ and summed over $i$ and $j$, a typical term from the "second order corrections" can be estimated as

$$
\begin{aligned}
\sum_{i, j}\left(\Delta x D_{x}^{+} D_{x}^{-} B_{i, j+1}^{1} D_{y}^{+}\left|u_{i, j}^{1}\right|_{\delta}\right) d_{i, j}=-\sum_{i, j} \Delta x\left(D_{x}^{-} B_{i, j+1}^{1}\right) D_{x}^{-}\left(D_{y}^{+}\left|u_{i, j}^{1}\right|_{\delta} d_{i, j}\right) \\
=-\sum_{i, j} \Delta x\left(D_{x}^{+} B_{i, j}^{1}\right)\left(\left(D_{x}^{-} D_{y}^{+}\left|u_{i, j}^{1}\right|_{\delta}\right) d_{i, j}+D_{y}^{+}\left|u_{i-1, j}^{1}\right|_{\delta} D_{x}^{-} d_{i, j}\right) \\
\leq\left\|\partial_{x y}^{2} u^{1}\right\|_{L^{\infty}\left(\mathbb{R}^{2}\right)} \sum_{i, j} 4\left(B_{i, j}^{1}\right)^{2}+2 d_{i, j}^{2}+\left\|\partial_{y} u^{1}\right\|_{L^{\infty}\left(\mathbb{R}^{2}\right)} \sum_{i, j} \frac{\Delta x}{4 \varepsilon}\left(D_{x}^{+} B_{i, j}^{1}\right)^{2}+\Delta x \varepsilon\left(D_{x}^{-} d_{i, j}\right)^{2} .
\end{aligned}
$$

Applying this to all the terms in the second order corrections, we get

$\sum_{i, j}$ (second order corrections) $2 d_{i, j} \leq 2\left(\left\|\partial_{x y}^{2} u^{1}\right\|_{L^{\infty}\left(\mathbb{R}^{2}\right)}+\left\|\partial_{x x}^{2} u^{1}\right\|_{L^{\infty}\left(\mathbb{R}^{2}\right)}+\left\|\partial_{x y}^{2} u^{2}\right\|_{L^{\infty}\left(\mathbb{R}^{2}\right)}+\left\|\partial_{y y}^{2} u^{2}\right\|_{L^{\infty}\left(\mathbb{R}^{2}\right)}\right)$

$$
\begin{aligned}
& \times \sum_{i, j}\left(4 \mathbf{B}_{i, j}^{2}+2 d_{i, j}^{2}\right)+4\left(\left\|\partial_{x} u^{1}\right\|_{L^{\infty}\left(\mathbb{R}^{2}\right)}+\left\|\partial_{y} u^{1}\right\|_{L^{\infty}\left(\mathbb{R}^{2}\right)}+\left\|\partial_{x} u^{2}\right\|_{L^{\infty}\left(\mathbb{R}^{2}\right)}+\left\|\partial_{y} u^{2}\right\|_{L^{\infty}\left(\mathbb{R}^{2}\right)}\right) \\
& \times \sum_{i, j} \frac{1}{4 \varepsilon}\left(\Delta x\left(D_{x}^{-} B_{i, j}^{1}\right)^{2}+\Delta y\left(D_{y}^{-} B_{i, j}^{2}\right)^{2}\right)+\varepsilon\left(\Delta x\left(D_{x}^{-} d_{i, j}\right)^{2}+\Delta y\left(D_{y}^{-} d_{i, j}\right)^{2}\right)
\end{aligned}
$$

Now the first terms on the right of (2.36) will save the day. When multiplied by $2 d_{i, j}$, the first of these yields

$$
\begin{aligned}
\sum_{i, j} \Delta x d_{i, j}\left|u_{i, j}^{1}\right|_{\delta} D_{x}^{+} D_{x}^{-} d_{i, j} & =-\sum_{i, j} \Delta x D_{x}^{-}\left(d_{i, j}\left|u_{i, j}^{1}\right|_{\delta}\right) D_{x}^{-} d_{i, j} \\
& =-\sum_{i, j} \Delta x\left|u_{i, j}^{1}\right|_{\delta}\left(D_{x}^{-} d_{i, j}\right)^{2}+\Delta x d_{i-1, j} D_{x}^{-}\left|u_{i, j}^{1}\right|_{\delta} D_{x}^{-} d_{i, j} \\
& \leq-\sum_{i, j} \Delta x\left|u_{i, j}^{1}\right|_{\delta}\left(D_{x}^{-} d_{i, j}\right)^{2}+5\left\|\partial_{x} u^{1}\right\|_{L^{\infty}\left(\mathbb{R}^{2}\right)} \sum_{i, j} d_{i, j}^{2} .
\end{aligned}
$$

Similarly we have that

$$
\sum_{i, j} \Delta y d_{i, j}\left|u_{i, j}^{2}\right|_{\delta} D_{y}^{+} D_{y}^{-} d_{i, j} \leq-\sum_{i, j} \Delta x\left|u_{i, j}^{2}\right|_{\delta}\left(D_{y}^{-} d_{i, j}\right)^{2}+4\left\|\partial_{y} u^{2}\right\| \sum_{i, j} d_{i, j}^{2} .
$$


Collecting all our bounds, we find that there are finite constants $C_{\ell}, \ell=1, \ldots, 4$, depending only on $u^{1}, u^{2}$ and their first and second derivatives, such that

$$
\begin{aligned}
\frac{\mathrm{d}}{\mathrm{d} t} \sum_{i, j} d_{i, j}^{2} \leq & C_{1} \sum_{i, j} d_{i, j}^{2}+C_{2} \sum_{i, j} \mathbf{B}_{i, j}^{2}+C_{3} \frac{1}{\varepsilon} \sum_{i, j} \Delta x\left(D_{x}^{-} B_{i, j}^{1}\right)^{2}+\Delta y\left(D_{y}^{-} B_{i, j}^{2}\right)^{2} \\
& +\left(C_{4} \varepsilon-\frac{\delta}{2}\right) \sum_{i, j}\left(\Delta x\left(D_{x}^{-} d_{i, j}\right)^{2}+\Delta y\left(D_{y}^{-} d_{i, j}\right)^{2}\right) .
\end{aligned}
$$

Remembering that $\delta$ is a fixed positive number, we now choose $\varepsilon$ positive, but so small that $\left(C_{4} \varepsilon-\delta / 2\right) \leq 0$. Next use the estimates (2.16) and (2.14), multiply by $\Delta x \Delta y$ and integrate over the interval $(0, t)$ to get the divergence bound:

Lemma 2.3. Assume that $\mathbf{u} \in C^{2}\left(\mathbb{R}^{2}\right)$. Let $\left\{\mathbf{B}_{i, j}(t)\right\}$ be defined by (2.7) and define the discrete divergence

$$
d_{i, j}^{n}(t)=D_{x}^{0}\left(B_{i, j}^{1}(t)\right)+D_{y}^{0}\left(B_{i, j}^{2}(t)\right) .
$$

Then

$$
\Delta x \Delta y \sum_{i, j}\left(d_{i, j}(t)\right)^{2} \leq \mathrm{e}^{C t}\left(\Delta x \Delta y \sum_{i, j}\left|d_{i, j}(0)\right|^{2}+\Delta x \Delta y \sum_{i, j}\left|\mathbf{B}_{i, j}(0)\right|^{2}\right)
$$

for some finite constant $C$ depending only on $\delta$ and on $\mathbf{u}$ and its derivatives up to second order.

Remark 2.2. If the limit of the scheme, $\mathbf{B}$, has bounded first and second derivatives, and the approximate solution $\mathbf{B}^{\Delta x}$ has uniformly (in $\Delta x$ and $\Delta y$ ) bounded first and second differences, then the terms hidden behind "discrete correction terms" and "terms from $D^{+} D^{-}$" in (2.29) will be $\mathcal{O}(\Delta x)+\mathcal{O}(\Delta y)$. Hence if $\operatorname{div}(\mathbf{B}(x, y, 0))=0$, also $\operatorname{div}(\mathbf{B}(x, y, t))=0$ for $t>0$. Furthermore,

$$
\operatorname{div}^{0}\left(\mathbf{B}^{\Delta x}(x, y, t)\right)=\mathcal{O}(\Delta x)+\mathcal{O}(\Delta y) .
$$

\section{NumericAl EXAMPLES}

In this section, we test the stable upwind scheme (SUS) (2.8) and compare it with other schemes. We present test cases where we compare the performance of SUS with the standard upwind scheme (1.19), and with the scheme of Torrilhon and Fey (1.20), which we henceforth refer to as the TF scheme.

\subsection{Example 1}

The first example uses

$$
\mathbf{u}=(1,2), \quad \text { and } \quad B_{0}^{1}(x, y)=B_{0}^{2}(x, y)= \begin{cases}2 & \text { if } x>y \\ 0 & \text { otherwise }\end{cases}
$$

Since $\mathbf{u}$ is constant, the exact solution is

$$
\mathbf{B}(x, y, t)=\mathbf{B}_{0}(x-t, y-2 t) .
$$

As a computational domain we use $(x, y) \in[-0.5,0.5]^{2}$ and Neumann boundary conditions. All schemes we tested used a CFL-number of $1 / 2$. In Figure 1 we show how some schemes compute the approximation at $t=0.3$, with $\Delta x=\Delta y=0.01$. We see that the TF scheme seems to be more accurate than the SUS scheme, at the expense of some oscillations as the solutions generated with the TF scheme are not TVD even in this simple case of constant velocity fields. The standard upwind scheme, (1.19), gives a solution similar to SUS, 
TABLE 1. Relative conservation errors for the SUS scheme.

\begin{tabular}{c|cccccc}
\hline & $\Delta x=2^{-6}$ & $\Delta x=2^{-7}$ & $\Delta x=2^{-8}$ & $\Delta x=2^{-9}$ & $\Delta x=2^{-10}$ & $\Delta x=2^{-11}$ \\
\hline SUS & 1.4577 & 0.7774 & 0.4019 & 0.2044 & 0.1031 & 0.0518 \\
\hline
\end{tabular}

but with one pronounced spike. The scheme called "central Powell" is the result of central evaluation of the Powell source term, and is seen to be unstable. This example shows that the Godunov-Powell source term has to be introduced as well as upwinded in some manner in order to get stable results. The SUS scheme relies on an implicit upwinding due to the use of the Friedrichs form.

\subsection{Example 2}

The goal of this example is to investigate how the discrete divergence introduced by the SUS scheme varies with $\Delta t$, as well as to compare the SUS scheme with the TF scheme.

In order to do this we choose divergence free initial data given by

$$
B_{0}^{1}(x, y)=\partial_{y} A(x, y), B_{0}^{2}(x, y)=-\partial_{x} A(x, y), \text { where } A=\frac{1}{2 \pi} \sin (2 \pi x) \sin (2 \pi y)+y-x,
$$

and

$$
\mathbf{u}=(1,1)+0.25(\cos (2 \pi x)+2 \sin (2 \pi y), \sin (2 \pi x)+2 \cos (2 \pi y)) .
$$

To compute approximations we use periodic boundary conditions in the domain $(x, y) \in[-0.5,0.5]^{2}$ and $t \in$ $[0,0.5]$. Although in violation of the CFL-condition (2.12), both the SUS and the TF scheme use the CFLnumber $1 / 2$. Figure 2 shows how the discrete divergence(s) for both schemes vary with $\Delta x$. We have not shown div $^{*}$ for the TF scheme, since this remains very small throughout the computation. We have used $\Delta x=\Delta y=2^{-6}, 2^{-7}, \ldots, 2^{-11}$. From Figure 2 we see that both $\operatorname{div}^{0}$ and $\operatorname{div}^{*}$ seem to converge to zero for the SUS scheme at the expected first order accuracy, but $\operatorname{div}^{0}$ does not seem to converge to zero for the TF scheme, although this scheme preserves div $^{*}$ to machine precision. This example indicates that although a scheme preserves some discrete divergence, other discrete divergences need not be small.

Since the SUS-scheme proposed here is not conservative, it is interesting to see how much of $\mathbf{B}$ is lost. We have measured the relative conservation error defined as

$$
100 \times \sum_{i=1}^{2} \frac{\left|\iint B^{i, \Delta t}(x, y, t)-B^{i, \Delta t}(x, y, 0) \mathrm{d} x \mathrm{~d} y\right|}{\left|\iint B^{i, \Delta t}(x, y, 0) \mathrm{d} x \mathrm{~d} y\right|}
$$

From Table 1, it is clear that although some mass is lost due to the fact that SUS scheme is not conservative, the errors are quite small and converge to zero as the mesh is refined at the expected first order of accuracy.

\subsection{Example 3}

If $\mathbf{u}(x, y)=(-y, x)$, i.e., a rotation around the origin, the solution to (1.3) in two space dimensions is given by

$$
\mathbf{B}(\mathbf{x}, t)=R(t) \mathbf{B}_{0}(R(-t) \mathbf{x}),
$$

where $R(t)$ is a rotation matrix for a rotation with angle $t$. This means that $\mathbf{B}(\mathbf{x}, 2 \pi)=\mathbf{B}_{0}(\mathbf{x})$, which makes comparisons between approximations and the exact solution easy. We have taken the initial data from [19],

$$
\mathbf{B}_{0}(x, y)=4\left(\begin{array}{c}
-y \\
x-\frac{1}{2}
\end{array}\right) \mathrm{e}^{-20\left((x-1 / 2)^{2}+y^{2}\right)} .
$$

Note that $\operatorname{div}\left(\mathbf{B}_{0}\right)=0$. We have used the computational domain $(x, y) \in \Omega=[-1,1]^{2}$ and Neumann boundary conditions, and compared the SUS and the TF schemes. In Table 2 we show the relative $L^{2}$ errors produced 

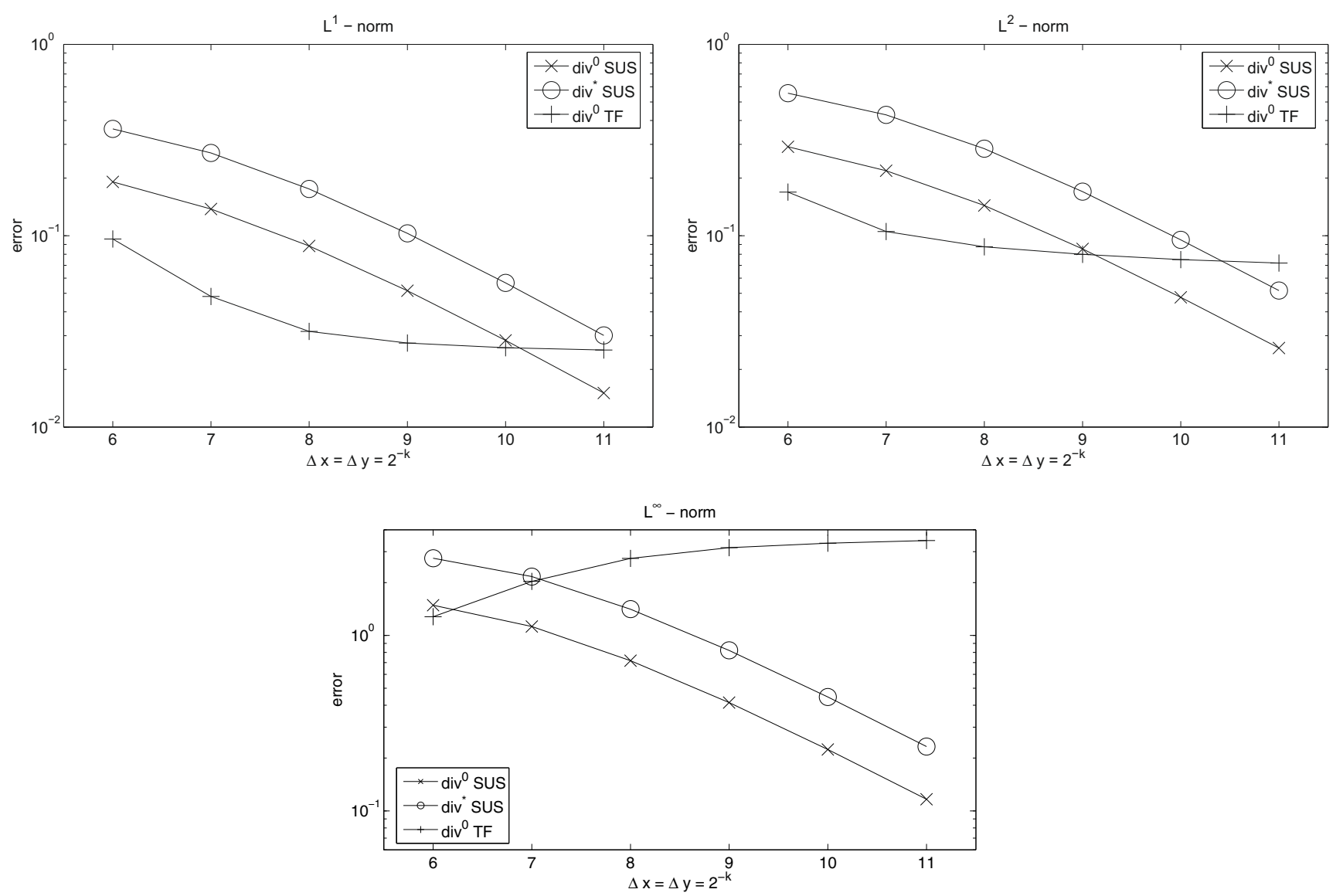

FIGURE 2. Discrete divergences for the SUS and the TF schemes for the approximate solutions as $t=0.5$, as a function of $\Delta x$. Top left: $L^{1}$ norm, top right: $L^{2}$ norm, left: $L^{\infty}$ norm.

TABLE 2. Relative errors for the SUS and TF schemes with initial data given by (3.2).

\begin{tabular}{l|cccccc}
\hline & $\Delta x=2^{-5}$ & $\Delta x=2^{-6}$ & $\Delta x=2^{-7}$ & $\Delta x=2^{-8}$ & $\Delta x=2^{-9}$ & $\Delta x=2^{-10}$ \\
\hline SUS & 79 & 61 & 42 & 26 & 15 & 8 \\
TF & 67 & 52 & 36 & 23 & 13 & 7 \\
\hline
\end{tabular}

by the schemes, for $\Delta x=\Delta y=2^{-5}, \ldots, 2^{-10}$, where both schemes used the CFL-number $1 / 2$. The relative error is defined as

$$
e=100 \times \frac{\left\|\mathbf{B}^{\Delta t}(\cdot, 2 \pi)-\mathbf{B}^{\Delta t}(\cdot, 0)\right\|_{L^{2}(\Omega)}}{\left\|\mathbf{B}^{\Delta t}(\cdot, 0)\right\|_{L^{2}(\Omega)}}
$$

Although we observed that the two schemes produced solutions which were different in some details, both schemes have comparable $L^{2}$ errors. Also the numerical convergence rate of both schemes is slightly less than one. Therefore, for a scheme to work reasonably well, it does not seem crucial that it preserves some discrete divergence. 

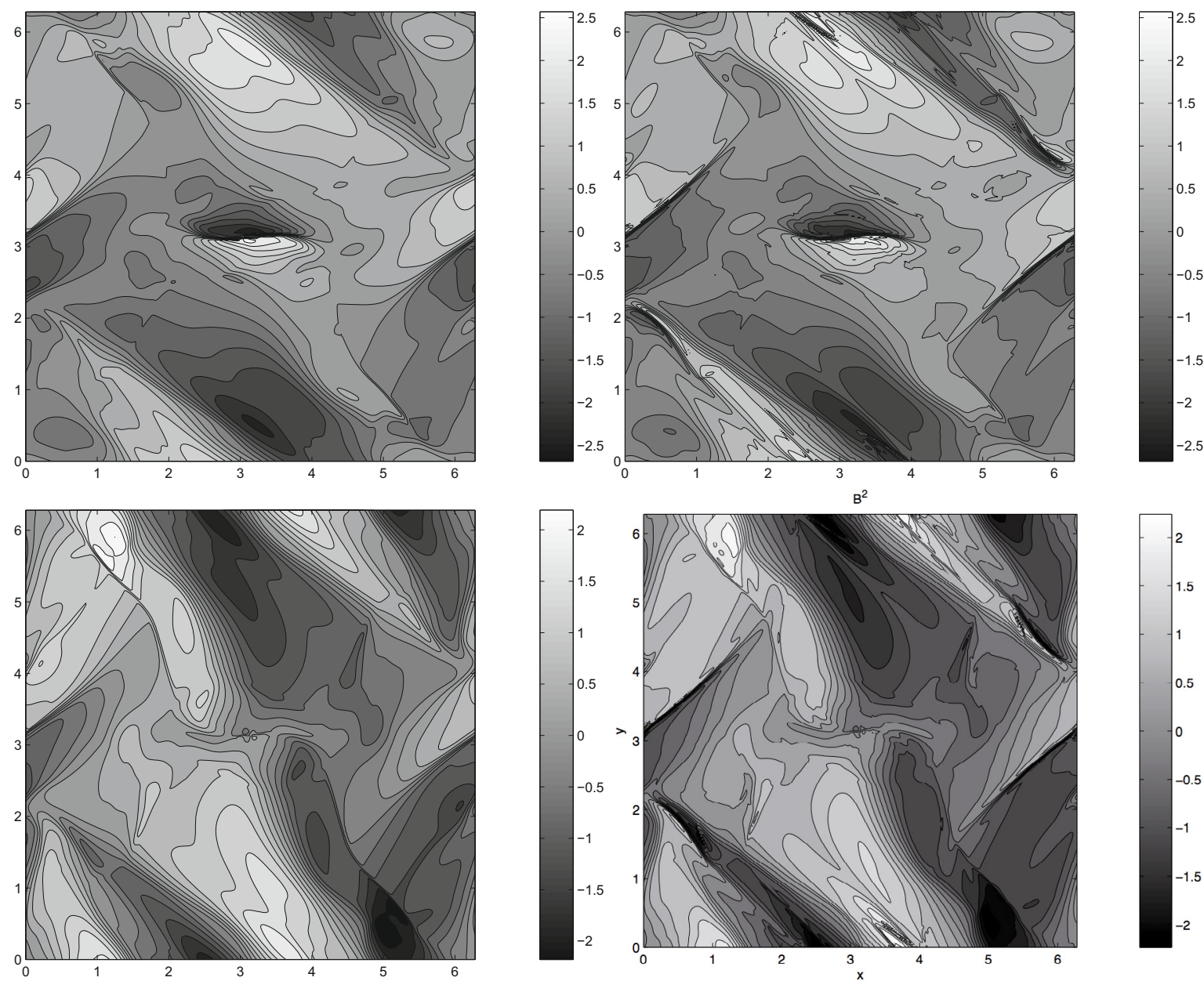

FigurE 3. The approximations at $t=\pi$, left column: SUS, right column: TF.

\subsection{Example 4}

The analysis presented in this paper can easily be extended to a time dependent velocity filed $\mathbf{u}=\mathbf{u}(\mathbf{x}, t)$. Therefore we present an example with a time dependent velocity field. This velocity field originates from a simulation of the well-known test case for magnetohydrodynamics called the "Orszag-Tang vortex", and is given as a $\operatorname{table}^{1} \mathbf{u}_{i, j}^{n}$.

We have used the computational domain $(x, y) \in[0,2 \pi]^{2}$ with periodic boundary conditions, and $t \in[0, \pi]$. The initial magnetic field is

$$
\mathbf{B}_{0}(x, y)=\left(\begin{array}{c}
-\sin (y) \\
\sin (2 x)
\end{array}\right)
$$

In this example, $\Delta t$ is not constant, and the time steps are given in the table. Although $\mathbf{u}$ is initially smooth, it develops shocks after some time steps. The test used $\Delta x=\Delta y=2 \pi / 300$. Figure 3 shows the approximations generated by the SUS and the TF schemes for this example. The solution is complicated, with many features, but we see that the schemes produce similar results. In Figure 4 we illustrate this by plotting the approximation to $B^{1}$ along the lines $y=(122 / 300) \times 2 \pi$ and $y=(293 / 300) \times 2 \pi$. From this figure, we see that the TF scheme

\footnotetext{
${ }^{1}$ This table can be downloaded from http://folk.uio.no/ franzf/OT300x300.tar.gz
} 
$B^{1}$

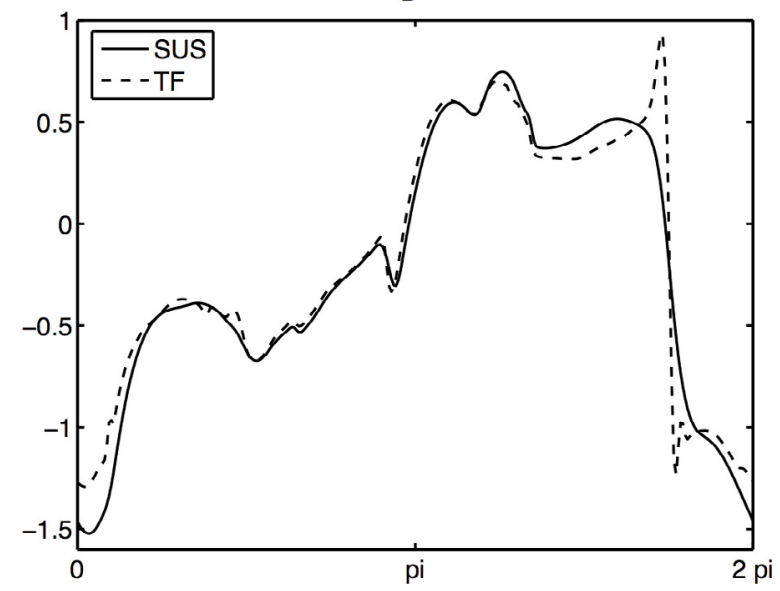

$B^{1}$

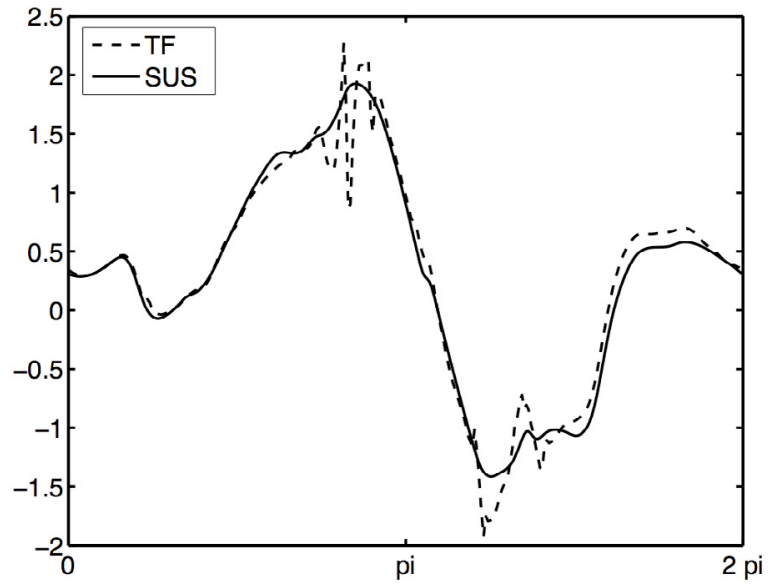

Figure 4. Left: $B^{1}(x, 2.54, \pi)$, right: $B^{1}(x, 6.13, \pi)$.

resolve discontinuities better, but since it is less stable, some of the "spikes" are numerical artifacts and reflect the lack of TVD property in the TF scheme. We should emphasize that even though our analysis requires that the velocity field $u$ is sufficiently smooth, we have found that the SUS scheme works very well even for non-smooth velocity fields.

\section{Conclusions}

Based on these numerical experiments, we conclude that the stable upwind scheme (2.8) proposed in this paper is robust, efficient and reasonably accurate compared with other first order schemes.

Regarding divergence preservation, the divergence errors generated by the scheme are small and converge to zero with $\Delta t$. Furthermore, two different discrete forms of the divergence are of the same magnitude. On the other hand, even though the scheme of Torrilhon and Fey (1.20) preserves one form of discrete divergence, it does not preserve other discrete divergences. Therefore, we think that if a numerical scheme for (1.1) is stable, exact divergence preservation is not necessary to obtain good results.

We also remark that it is straightforward to extend our result to three space dimensions, i.e., $\mathbf{B}=\left(B^{1}, B^{2}, B^{3}\right)$ $(x, y, z)$, by upwinding the Godunov-Powell source term. We plan to extend the ideas presented here both to unstructured meshes and higher orders in a forthcoming paper.

\section{REFERENCES}

[1] D.S. Balsara and D. Spicer, A staggered mesh algorithm using high order Godunov fluxes to ensure solenoidal magnetic fields in magnetohydrodynamic simulations. J. Comp. Phys. 149 (1999) 270-292.

[2] T.J. Barth, Numerical methods for gas dynamics systems, in An introduction to recent developments in theory and numerics for conservation laws, D. Kröner, M. Ohlberger and C. Rohde Eds., Springer (1999).

[3] S. Benzoni-Gavage and D. Serre, Multidimensional hyperbolic, Partial differential equations - First-order systems and applications, Oxford Mathematical Monographs. The Clarendon Press, Oxford University Press, Oxford (2007).

[4] N. Besse and D. Kröner, Convergence of the locally divergence free discontinuous Galerkin methods for induction equations for the 2D-MHD system. ESAIM: M2AN 39 (2005) 1177-1202.

[5] J.U. Brackbill and D.C. Barnes, The effect of nonzero div $B$ on the numerical solution of the magnetohydrodynamic equations. J. Comp. Phys. 35 (1980) 426-430.

[6] W. Dai and P.R. Woodward, A simple finite difference scheme for multi-dimensional magnetohydrodynamic equations. J. Comp. Phys. 142 (1998) 331-369.

[7] C. Evans and J.F. Hawley, Simulation of magnetohydrodynamic flow: a constrained transport method. Astrophys. J. 332 (1998) 659. 
[8] F.G. Fuchs, S. Mishra and N.H. Risebro, Splitting based finite volume schemes for ideal MHD equations. J. Comp. Phys. 228 (2009) 641-660.

[9] S.K. Godunov, The symmetric form of magnetohydrodynamics equation. Num. Meth. Mech. Cont. Media 1 (1972) 26-34.

[10] J.D. Jackson, Classical Electrodynamics. Third Edn., Wiley (1999).

[11] V. Jovanovič and C. Rohde, Finite volume schemes for Friedrichs systems in multiple space dimensions: a priori and a posteriori error estimates. Num. Meth. PDE 21 (2005) 104-131.

[12] R.J. LeVeque, Finite volume methods for hyperbolic problems. Cambridge University Press (2002).

[13] G.K. Parks, Physics of Space Plasmas: An Introduction. Addition-Wesley (1991).

[14] K.G. Powell, A approximate Riemann solver for magnetohydrodynamics (that works in more than one dimension). Technical report 94-24, ICASE, Langley, VA, USA (1994).

[15] K.G. Powell, P.L. Roe, T.J. Linde, T.I. Gombosi and D.L. De Zeeuw, A solution adaptive upwind scheme for ideal MHD. J. Comp. Phys. 154 (1999) 284-309.

[16] J. Rossmanith, A wave propagation method with constrained transport for shallow water and ideal magnetohydrodynamics. Ph.D. Thesis, University of Washington, Seattle, USA (2002).

[17] D.S. Ryu, F. Miniati, T.W. Jones and A. Frank, A divergence free upwind code for multidimensional magnetohydrodynamic flows. Astrophys. J. 509 (1998) 244-255.

[18] M. Torrilhon, Locally divergence preserving upwind finite volume schemes for magnetohyrodynamic equations. SIAM. J. Sci. Comp. 26 (2005) 1166-1191.

[19] M. Torrilhon and M. Fey, Constraint-preserving upwind methods for multidimensional advection equations. SIAM. J. Num. Anal. 42 (2004) 1694-1728.

[20] G. Toth, The $\operatorname{div} B=0$ constraint in shock capturing magnetohydrodynamics codes. J. Comp. Phys. 161 (2000) 605-652.

[21] J-P. Vila and P. Villedeau, Convergence of explicit finite volume scheme for first order symmetric systems. Numer. Math. 94 (2003) 573-602. 